\title{
Folate-Conjugated Chitosan-Pluronic P123 Nanogels: Synthesis and Characterizations towards Dual Drug Delivery
}

\author{
Van Toan Nguyen, ${ }^{1,2}$ Thi Hương Nguyen, ${ }^{3}$ Le Hang Dang, ${ }^{4}$ Hieu Vu-Quang ${ }^{\circ},{ }^{5}$ \\ and Ngoc Quyen Tran (iD) 2,4 \\ ${ }^{1}$ Institute of Research and Development, Duy Tan University, Da Nang City 550000, Vietnam \\ ${ }^{2}$ Graduate University of Science and Technology, Vietnam Academy of Science and Technology, Ho Chi Minh City 700000, Vietnam \\ ${ }^{3}$ Institute of Chemistry and Material, Hanoi City 100000, Vietnam \\ ${ }^{4}$ Institute of Applied Materials Science, Vietnam Academy of Science and Technology, Ho Chi Minh City 700000, Vietnam \\ ${ }^{5}$ NTT Hi-Tech Institute, Nguyen Tat Thanh University, Ho Chi Minh City 700000, Vietnam
}

Correspondence should be addressed to Hieu Vu-Quang; vqhieu@ntt.edu.vn and Ngoc Quyen Tran; tnquyen979@gmail.com

Received 16 July 2019; Revised 16 October 2019; Accepted 1 November 2019; Published 21 December 2019

Academic Editor: Hassan Karimi-Maleh

Copyright (c) 2019 Van Toan Nguyen et al. This is an open access article distributed under the Creative Commons Attribution License, which permits unrestricted use, distribution, and reproduction in any medium, provided the original work is properly cited.

In this study, a self-assembled nanogel-based pluronic P123-grafted chitosan-folate (CP-FA) was fabricated as a paclitaxel/curcumin codelivery system. ${ }^{1} \mathrm{H}-\mathrm{NMR}$ and TGA proved that the fabricating method of CP-FA was successful. Dynamic light scattering (DLS), zeta potentials, and transmission electron microscopy (TEM) exposed that CP-FA nanoparticles had a uniform size with a diameter of around $16.27 \pm 2.01 \mathrm{~nm}$ in the colloidal solution and had better sustainable stability at a lower concentration than P123 due to the moderate positive potential value $(39.43 \pm 3.45 \mathrm{mV})$ and the lower critical micelle concentration $(0.036 \mathrm{mg} / \mathrm{ml})$. Dual drugs were loaded with CP-FA nanogels via self-assembly by the hydrophobic interaction between both hydrophobic therapeutic compounds (PTX and Cur) and the hydrophobic segment of the P123 copolymer. The high hydrophobicity of the segment induced a great loading efficacy of up to $98.63 \pm 0.42$ of PTX and $97.82 \pm 0.48$ of Cur. In addition, the CP-FA nanogels exposed superior effects in a controlled release of these encapsulated therapeutic compounds for a long period of time. The anticancer activity of the dual-drug delivery system was evaluated using human breast cancer cell lines (MCF-7) via the IC50 value to compare with the PTX-loading CP-FA nanogel. The obtained results suggested that CP-FA/PTXCur displayed a remarkable improvement in anticancer activity at an IC50 value of $5.74 \pm 0.23 \mathrm{nM}$ which was higher than that of CP-FA/PTX (IC50 = 8.20 $\pm 1.41 \mathrm{nM})$ due to the synergistic effect of both PTX and Cur. Thereby, a dual-drug deliverysystem-based CP-FA of PTX and Cur has been proposed as a promising candidate in cancer therapy.

\section{Introduction}

In the past few decades, polysaccharide-based amphiphilic copolymers have been widely studied as drug and gene delivery systems due to their specific interactions with several kinds of bioactive molecules $[1,2]$. Through the orientation of distinctive segments of these amphiphilic copolymers in aqueous solution, their self-assembly models induce various desirable core-shell platforms with a homogeneous particle size. The most substantial advances of core-shell nanoparticles are the ideal location in which hydrophobic agents such as chemotherapy drugs (cisplatin, paclitaxel, etc.), bioactive compounds (curcumin, quercetin, etc.), fluorescent probes (pyrazole, etc.), or genes/proteins can be protected by the hydrophilic segments organizing into the shell, whereas the pharmacokinetics of this compound become more significant [3-7]. Along with the advantage of a core-shell nanostructure, the introduction of natural polymers such as chitosan, heparin, or hyaluronic acid also helps to protect nanoparticles from opsonization due to the imparting of a stealth effect on the shell [8-10]. Moreover, because of the nature of the polysaccharide, the polysaccharide- 
based amphiphilic copolymers could improve the biocompatibility of the nanocarriers as well as enhance the interaction with biological entities such as the surface protein of the cell membrane leading to a viable drug delivery strategy.

Among amphiphilic copolymers, pluronics are wellknown as biocompatible polymers. The range application of pluronics has been expanding to include various therapeutic formulations in both academic and industrial fields. Several pluronics are listed in U.S. pharmacopoeia and are approved for various medical uses as excipients [11-13]. Among them, pluronic P123 (P123) is particularly appropriate as a carrier for water-insoluble drugs, e.g., paclitaxel, docetaxel, and curcumin $[14,15]$. Due to the low value of CMC $\left(4.4 \times 10^{-6} \mathrm{~mol} / \mathrm{l}\right)$ and a higher ratio of hydrophobic segments (hydrophilic-lipophilic balance, HLB = 8), P123 provides a strong capacity for hydrophobic drug loading [16]. Despite its effectiveness in the entrapment of hydrophobic drugs, the use of P123 as a carrier is still unpopular due to a potential dissolution of the cell membrane [15-17]. To eliminate the remaining problem of P123 related to its solubility and biological compatibility, P123 is usually fabricated with other drugs analogous to P123 with higher CMC and HLB values [15, 17]. For example, pharmacokinetic and biodistribution studies demonstrated that PTX-loaded P123/F127 could significantly increase the blood circulation time of PTX as compared to PTX loading P123 alone [18]. As another strategy to overcome the above problem, the modification of polysaccharides has recently been a popular approach [16]. Through a grafting method, the pluronic-conjugated polysaccharide resulted in the increased bioavailability of the carrier along with a high capacity for drug packaging. A pluronic F127grafted chitosan (CP) was successfully published in our previous study [16]. A colloidal dispersion of pluronic F127-grafted chitosan was under a core-shell structure with good biocompatibility, an immense capacity for packaging drugs, as well as excellent stability of micelles [19-23]. In addition, a chemically modified pluronic F127 with heparin [24] was successfully developed for a dual-drug delivery system in which the synergistic anticancer efficacy of curcumin and cisplatin was well-displaced in in vivo experiments [25]. However, despite the positive results of pluronic F127-grafted polysaccharide nanogels in drug delivery, there was a lack of reports related to this approach to the remaining problem of P123 in a drug delivery system. Moreover, by using an appropriate nanocarrier, the synergistic effect against cancer cell growth may improve effectiveness in cancer treatment [26]. Gao et al. [27] established that the codelivery of PTX and Cur in single-carrier-based MPEG-PCL was a powerful strategy for cancer treatment as compared to a single Cur or PTXloaded micelles at the same dose. The combination of Cur and PTX in PLGA-phospholipid nanoparticles [28] or PEG-PNB-TC nanoformulation [29] strongly supports this point. Based on the aforementioned backgrounds, a cotherapy using Cur and PTX can not only palliate drug resistance but also amplify their anticancer capacity by reason of a synergistic therapeutic effect [27-29].
In this study, a new amphiphilic folate-conjugated chitosan-pluronic P123 nanogel was developed as an effective drug carrier system. It was proposed, therefore, that the use of a CP-FA nanocarrier to codeliver PTX and curcumin (Cur) would lead to an improvement in drug efficacy. The incorporation of a new amphiphilic CP copolymer with the synergistic PTX and Cur drugs could be a potential dualdrug delivery system in the future.

\section{Materials and Methods}

2.1. Materials. Chitosan from shrimp shells with low-viscosity (code 50494-25G-F), pluronic P123 (P123, 5.800 MW), folic acid, curcumin, and paclitaxel were purchased from SigmaAldrich. 3-Amino-1-propanol (Ami), p-nitrophenyl chloroformate (NPC), 1-ethyl-3-3-dimethyl aminopropyl carbodiimide (EDC), and N-hydroxysuccinimide (NHS) were supplied by Acros Organics. Solvents were obtained from Scharlau's Chemicals. Cellulose dialysis membranes (MWCO $14 \mathrm{kDa}$ and MWCO $3.5 \mathrm{kDa}$ ) were bought from Spectrum Laboratories (USA).

\subsection{Methods}

2.2.1. Synthesis of Folate-Conjugated Chitosan-Pluronic P123 Nanogels. Folate-conjugated chitosan-pluronic P123 (CPFA) was prepared via four stages as presented in Figure 1. P123 pluronic was first activated by NPC to obtain NPCP123-NPC. Then, NPC-P123-NPC was partially substituted with Ami to produce NPC-P123-OH, followed by conjugation with chitosan to obtain an amphiphilic CP copolymer through carbamate formation. In the final stage, folic acid was conjugated with a CP copolymer using a carbodiimide coupling reagent.

(1) Synthesis of NPC-P123-NPC. P123 (15 gram, $2.59 \mathrm{mmol})$ was activated with NPC (1.06 gram, $5.31 \mathrm{mmol})$ at $65^{\circ} \mathrm{C}$ under stirring. After $24 \mathrm{~h}$, the temperature of the reaction was reduced to R.T. before adding chloroform. Then, the product was collected by precipitation in a diethyl ether/hexane mixture $(1: 1 v / v)$. The obtained paste precipitant was dried in a vacuum oven to obtain NPC-P123-NPC.

(2) Synthesis of NPC-P123-OH. NPC-P123-NPC (12 gram, $1.94 \mathrm{mmol})$ was dissolved in chloroform solution $(1: 20 \mathrm{w} / \mathrm{v})$. Then, the solution of Ami $(2.04 \mathrm{mmol})$ in chloroform $(1: 20 v / v)$ was added dropwise into the NPC-P123-NPC solution (molar ratio of NPC-P123-NPC and Ami was $1: 1.05)$. After 24 hours, the resultant was precipitated in a diethyl ether/hexane mixture $(1: 1 v / v)$. The paste precipitant was dried under vacuum condition to obtain NPC-P123-OH.

(3) Synthesis of Chitosan-Grafted P123 (CP). Chitosan (100 mg) was dissolved in $20 \mathrm{ml}$ of acidic media ( $\mathrm{pH} 5.0$ ). NPC-P123-OH (1 gram) was dissolved in $20 \mathrm{ml}$ of ethanol and then added dropwise into the chitosan solution under stirring at room temperature for 24 hours. Finally, the product was dialyzed against cold distilled water using a 


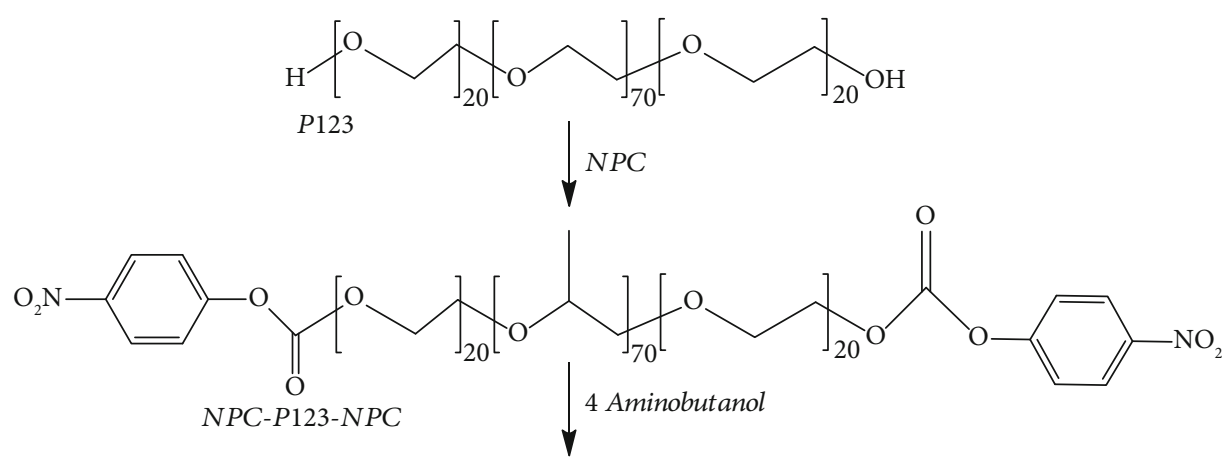<smiles>CC(OC(C)C(C)OCC(C)(C)OCC(C)(C)OC(=O)Oc1ccc([N+](=O)[O-])cc1)C(=O)NCCCO</smiles>

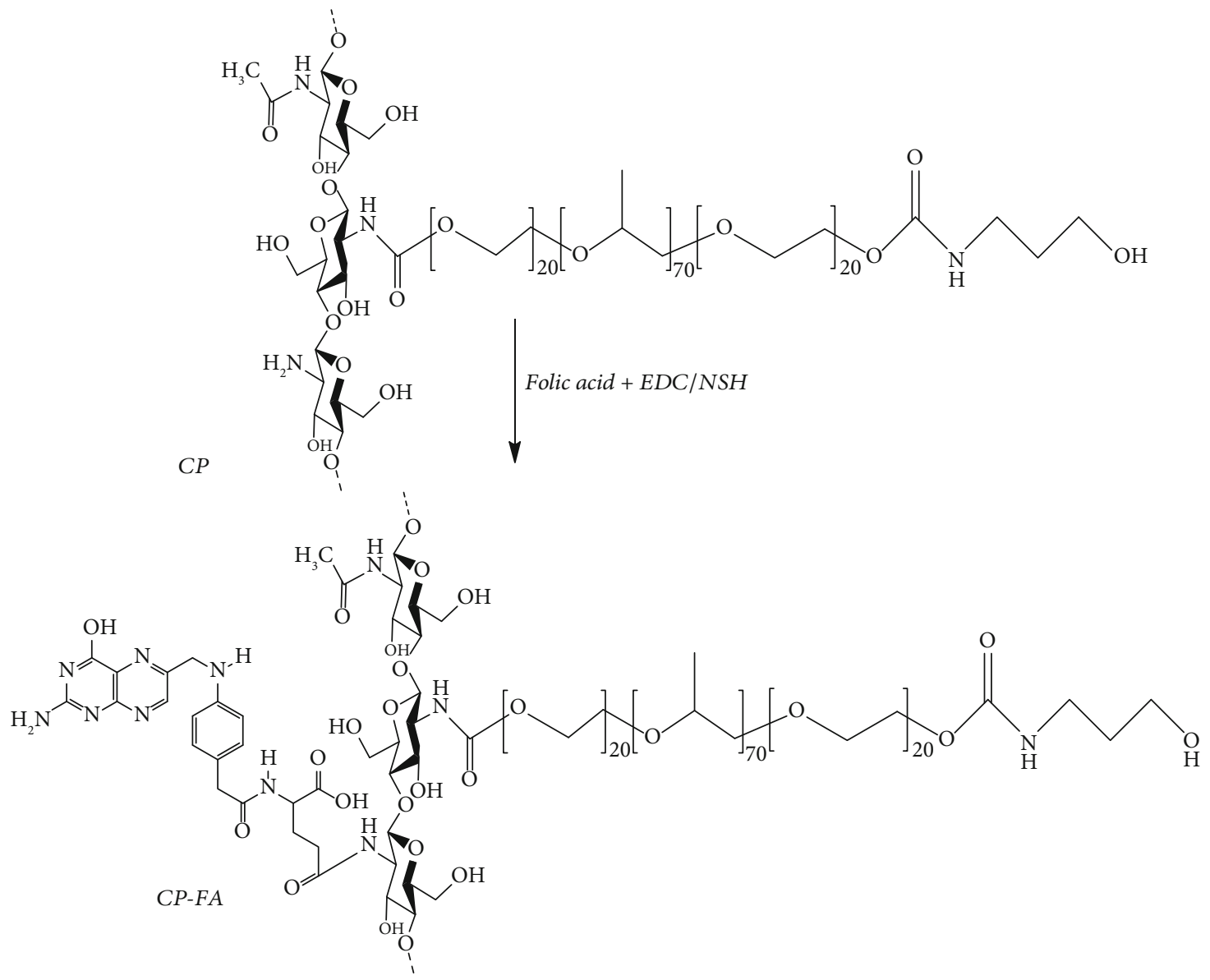

Figure 1: Preparation of chitosan-P123 copolymers.

cellulose membrane $(\mathrm{MWCO}=12 \mathrm{kDa})$ for 7 days and then lyophilized to obtain a powdery product of CP. To evaluate the loading ability of hydrophobic drugs, various feed weight ratios of P123 were conducted to graft onto chitosan (chitosan : 123 $=1: 5,1: 10,1: 15$, and $1: 20$ by mass).
(4) Synthesis of Folic Acid-Conjugated CP. A solution of folic acid was activated by EDC and NHS (molar ratio $=1: 1: 1$ ) in anhydrous DMSO (1 mg of folic acid/1 ml DMSO). The activated folic acid was then dropped slowly to a CP solution $(100 \mathrm{mg} / 1 \mathrm{ml} \mathrm{DI}$ water) and stirred at room temperature in the dark for $12 \mathrm{~h}$ to obtain a folate-conjugated $\mathrm{CP}$. Regarding 
the grafting degree of P123 onto chitosan (by TGA calculation), the molar ratio of folic acid and glucosamine used was used 1:2. Finally, the product was dialyzed against DI water for 7 days using a cellulose membrane $(\mathrm{MWCO}=12 \mathrm{kDa})$ and then lyophilized to obtain a powdery product of CP-FA. ${ }^{1} \mathrm{H}-\mathrm{NMR}$ (Bruker AMX-500) measurements were used to identify the chemical structure of copolymers. TGA (PerkinElmer TGA-7) was also used to assess compositions of the grafted copolymers.

2.2.2. Preparation and Characterizations of Nanogels. P123 micelles and CP nanogels were prepared in DI water. CMC values of the amphiphilic copolymers were determined via the iodine method using a UV-Visible spectrophotometer (Shimadzu UV-1700 PharmaSpec, Japan) at wavelengths from 300 to $400 \mathrm{~nm}$. The CMC values of the copolymer solution were calculated from the intersection of the two regression lines at which the vertical axis presented the absorption intensity, whereas the logarithm copolymer concentration was in a horizontal coordination [28]. Morphology and size distribution of the nanoparticles were characterized by transmission electron thermal stability microscopy (JEM-1400 JEOL) and dynamic light scattering (Nano Particle Analyzer SZ-100), respectively.

2.2.3. Preparation of Drug-Loaded Micelles (Nanogels) and Their Release Behavior. For drug encapsulation, a different amount of curcumin or PTX ethanol solution was added dropwise into $100 \mathrm{mg}$ carriers (CP or CP-FA) that were soluble in DI water under stirring condition. Ethanol solvent was evaporated to form drug-encapsulated nanogels via a hydrophobic interaction with the hydrophobic PPO blocks of P123. Subsequently, the mixtures were hydrated in $10 \mathrm{ml}$ of DI water and kept for 24 hours at RT to allow free drugs to settle down; this was followed by lyophilizing to obtain a powdery product of drug/copolymer mixtures. The amount of curcumin and/or PTX was determined by using UV absorbance at a wavelength of $420 \mathrm{~nm}$ and $254 \mathrm{~nm}$, respectively. The encapsulation efficiency percent (EE (\%)) and drug loading percent (DL (\%)) in the micelles were calculated using the following equation:

$$
\begin{aligned}
& \mathrm{EE}(\%)=\frac{\text { weight of the drug in micelles }}{\text { weight of the feeding drugs }} \times 100 \%, \\
& \mathrm{DL}(\%)=\frac{\text { weight of the drug in micelles }}{\text { weight of copolymers and drugs }} \times 100 \% .
\end{aligned}
$$

An in vitro drug release study was conducted by a diffusion method $[24,25]$. Briefly, four milligrams of a drug/copolymer was dispersed in $2 \mathrm{ml}$ of fresh phosphate-buffered saline (PBS; $\mathrm{pH}=7.4$ ) and then transferred into a dialysis bag $(\mathrm{MWCO}=3.5 \mathrm{kDa})$. Each dialysis bag was immersed in single vials containing $50 \mathrm{ml}$ of the release medium under mild shaking at $37^{\circ} \mathrm{C}$. For each sample, $5 \mathrm{ml}$ of the release medium was withdrawn at predetermined time intervals and replaced by the fresh medium at the same volume.
2.2.4. In Vitro Cytotoxicity. The cytotoxic effects of CP-FA, free PTX, PTX-loaded CP-FA (PTX-CP-FA), PTX/Curloaded CP-FA (PTX/Cur-CP-FA), and Cur-loaded CP-FA (Cur-CP-FA) were determined by the sulforhodamine $\mathrm{B}$ (SRB) colorimetric assay, using MCF-7 cells as the cancer cell model. All tested samples were first screened at $100 \mu \mathrm{g} / \mathrm{ml}$ before making a serial dilution in order to identify the halfmaximal inhibitory concentration (IC50) values. Briefly, MCF-7 cells were cultured in 96-well plates at a density of $10^{4}$ cells per well for $24 \mathrm{~h}$; then, various concentrations of drugs or its encapsulated nanocarriers were added, while the same volume of pure culture media was added into negative control wells. After $48 \mathrm{~h}$ of culture, all wells were fixed with $50 \%(w / v)$ trichloroacetic acid (Merck) solution for $1-3 \mathrm{~h}$ before being stained with $0.2 \%(w / v)$ SRB (SigmaAldrich) for $60 \mathrm{~min}$. $10 \mathrm{mM}$ Tris base solution (Promega) was applied after the unbound dye was removed by $1 \%$ acetic acid (Merck) (in $\mathrm{mM}$ ). The percentage of growth inhibition (\%I) was calculated according to the optical density values of $492 \mathrm{~nm}$ and $620 \mathrm{~nm}$ according to the following formula:

$$
\% I=\left(1-\frac{\mathrm{OD}_{\mathrm{t}}}{\mathrm{OD}_{\mathrm{c}}}\right) \times 100
$$

where $\mathrm{OD}_{t}$ and $\mathrm{OD}_{\mathrm{c}}$ are the optical density values of the test sample and the control sample, respectively. Camptothecin (Calbiochem) was used as a positive control. All trials were repeated at least 3 times $(n=3)$ and the data was presented as the mean \pm SD.

\section{Results and Discussions}

3.1. Characterization of the Activated P123, CP, and CP-FA. Figure 2(a) shows the ${ }^{1} \mathrm{H}-\mathrm{NMR}$ spectrum of NPC-P123NPC revealing the first success of the modified P123. Along with the proton signals on aromatic groups of NPC at $\delta \sim 8.3 \mathrm{ppm}$ (a) and $\delta \sim 7.4 \mathrm{ppm}$ (b), the proton of P123 was also exhibited. The resonance peak at $\delta \sim 3.6 \mathrm{ppm}$ (c) was characteristic of the protons on the $-\mathrm{CH}_{2}$ - group in the PEO block, whereas the resonance peaks at $\delta \sim 1.1$ ppm (d), $3.4 \mathrm{ppm}(\mathrm{e})$, and $3.5 \mathrm{ppm}$ (f) were characteristic of the protons of the $-\mathrm{CH}_{3},-\mathrm{CH}_{2}$, and $-\mathrm{CH}$ - groups on PPO chains, respectively. The successfully activated P123 was disclosed via the chemical shift at $\delta \sim 4.4 \mathrm{ppm}$ (g) according to the proton on the terminal methylene ester group on $\mathrm{CH}_{2}-\mathrm{O}-\mathrm{NPC}$. By using integral areas of the resonance peak at $\delta \sim 7.4 \mathrm{ppm}$ (b) and $1.1 \mathrm{ppm}(\mathrm{d})$, approximately $100 \%$ of the terminal hydroxyl groups were activated by NPC. The ${ }^{1} \mathrm{H}-\mathrm{NMR}$ spectrum of NPC-P123-OH also clearly shows resonance peaks characteristic of the protons of the P123 chain as in the spectrum of NPC-P123-NPC (Figure 2(b)). The appearance of the peak at $\delta \sim 4.2 \mathrm{ppm}$ (h) assigned to the methylene proton of Ami proved the success of the partially substituted step. From this spectrum, only $48.7 \%$ of the NPC groups remained in the NPC-P123-OH.

Grafting NPC-P123-OH onto a chitosan backbone was conducted at pH 5.0 to enhance the formation of the covalent bond between the NPC moiety and the amine groups on 


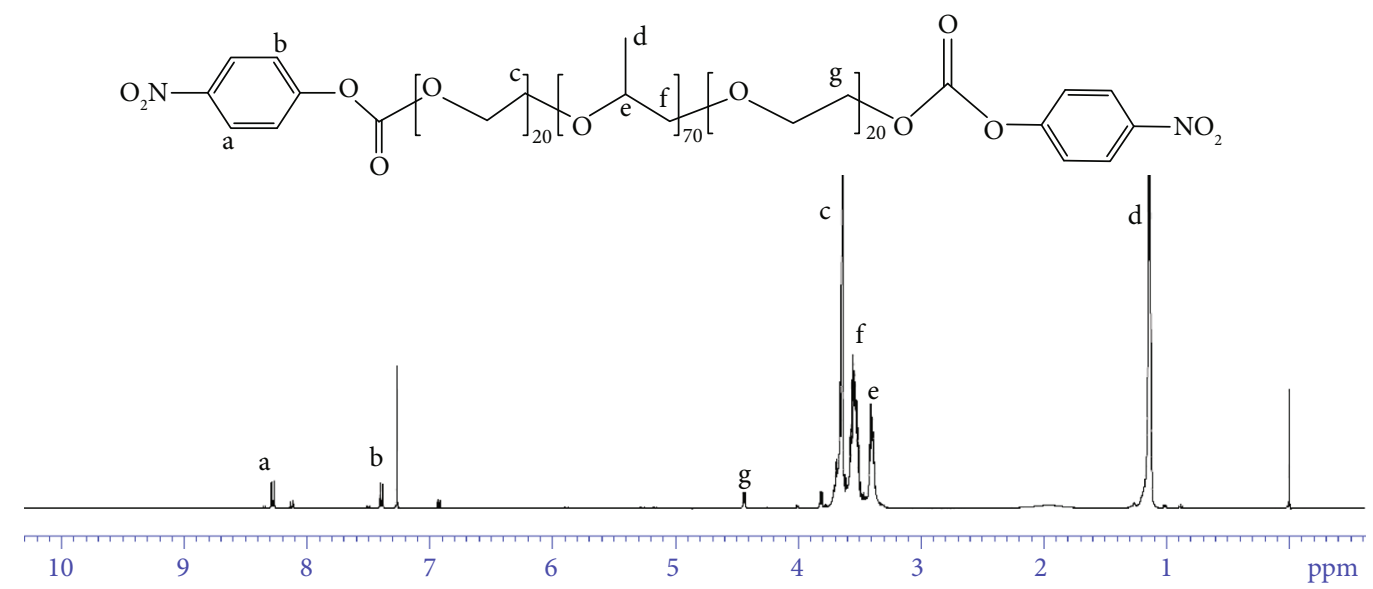

(a)

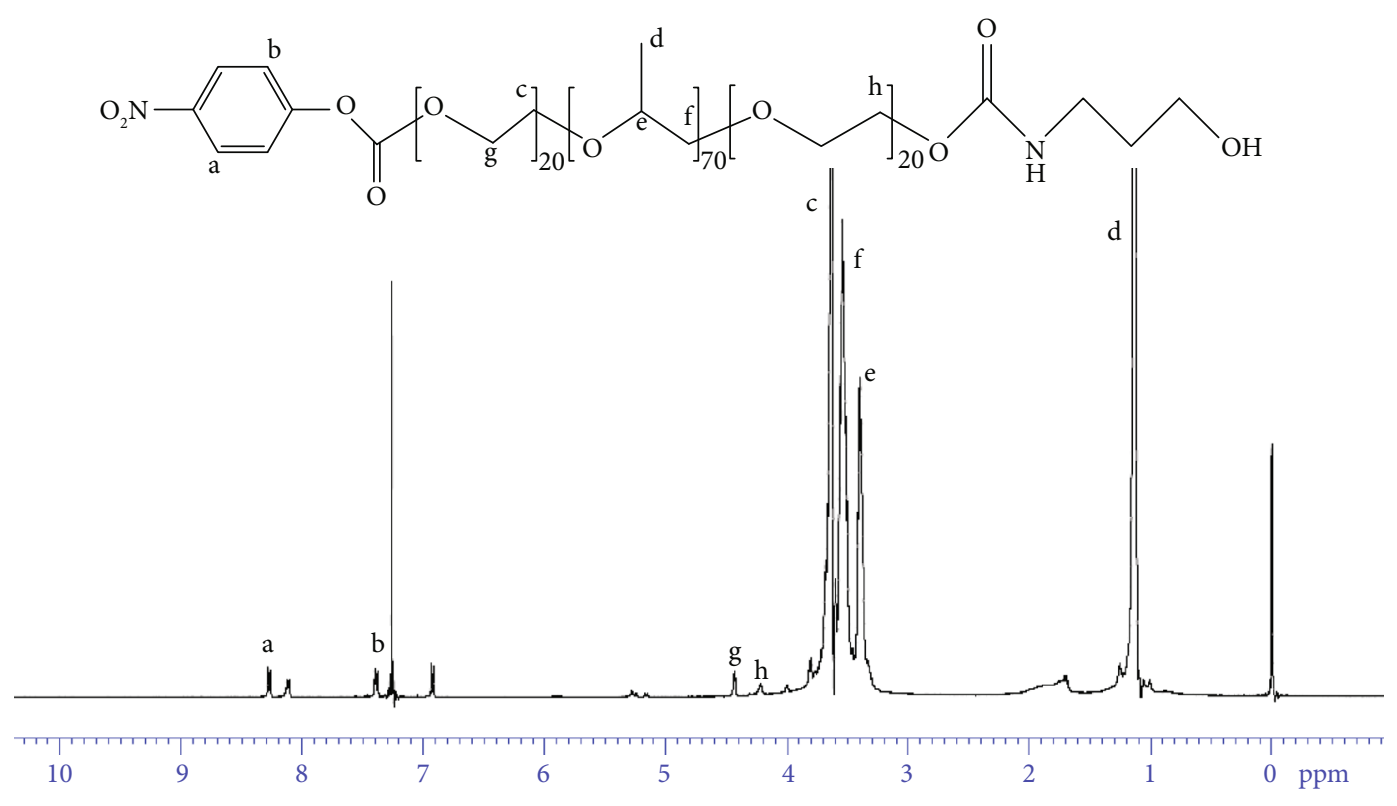

(b)

Figure 2: ${ }^{1} \mathrm{H}-\mathrm{NMR}$ spectra of NPC-P123-NPC (a) and NPC-P123-OH (b).

chitosan. The ${ }^{1} \mathrm{H}$-NMR spectrum of CP (Figure 3(a)) clearly exposes the resonance peaks of protons on $\mathrm{P} 123$ structures to accompany the appearance of two peaks at $\delta \sim 2.7 \mathrm{ppm}$ (i) and $\delta \sim 2.0 \mathrm{ppm}(\mathrm{j})$, which were assigned to protons at $\mathrm{C} 2$ carbon and the acetyl proton unit in chitosan. Besides, the disappearance of chemical signals of NPC at $\delta \sim 8.3 \mathrm{ppm}$ (a) and $\delta \sim 7.4$ ppm (b) indicated that P123 had been successfully grafted onto chitosan.

The ${ }^{1} \mathrm{H}-\mathrm{NMR}$ spectrum of CP-FA (Figure $3(\mathrm{~b})$ ) presented the appearance of three peaks at $\delta \sim 8.8 \mathrm{ppm}(\mathrm{m})$, $\delta \sim 7.4 \mathrm{ppm}(\mathrm{k})$, and $\delta \sim 6.7 \mathrm{ppm}(\mathrm{L})$, attributed to the proton resonance of the folate aromatic. The result could confirm that the CP-FA copolymer was obtained for targeted application towards the MCF-7 cancer cell in a further study.

The thermal stabilities of P123, chitosan, CP, and CP-FA were analyzed by TGA (Figure 4 ). The initial thermal decomposition of chitosan was at $100^{\circ} \mathrm{C}$ corresponding to the removal of absorbed water and following a main step at $250^{\circ} \mathrm{C}$. This further process happened slowly at $500^{\circ} \mathrm{C}$. P 123 exhibited one-step decomposition; the initial thermal decomposition point started at $340^{\circ} \mathrm{C}$ and almost completed weight loss at $420^{\circ} \mathrm{C}$. The TGA curves of $\mathrm{CP}(1: 10)$ contained features of both chitosan and P123 resulting in two steps of weight loss; the first was at $250^{\circ} \mathrm{C}$ inheriting from chitosan and rapid thermal decomposition at $340^{\circ} \mathrm{C}$ following the nature of P123. This patent was similar as other ratio grafting (the TGA curves of $\mathrm{CP}(1: 5), \mathrm{CP}(1: 15)$, and $\mathrm{CP}(1: 20)$ were not shown). The weight loss behavior of folic acid was partially similar to chitosan, but it had a lower thermal decomposition than that of chitosan. The initial thermal decomposition of folic acid was at $200^{\circ} \mathrm{C}$ and slowly decomposed according to the increase of the temperature. In addition, the TGA curves of CP-FA showed a slightly higher thermal stability as compared to $\mathrm{CP}$ in the range of 100 to 


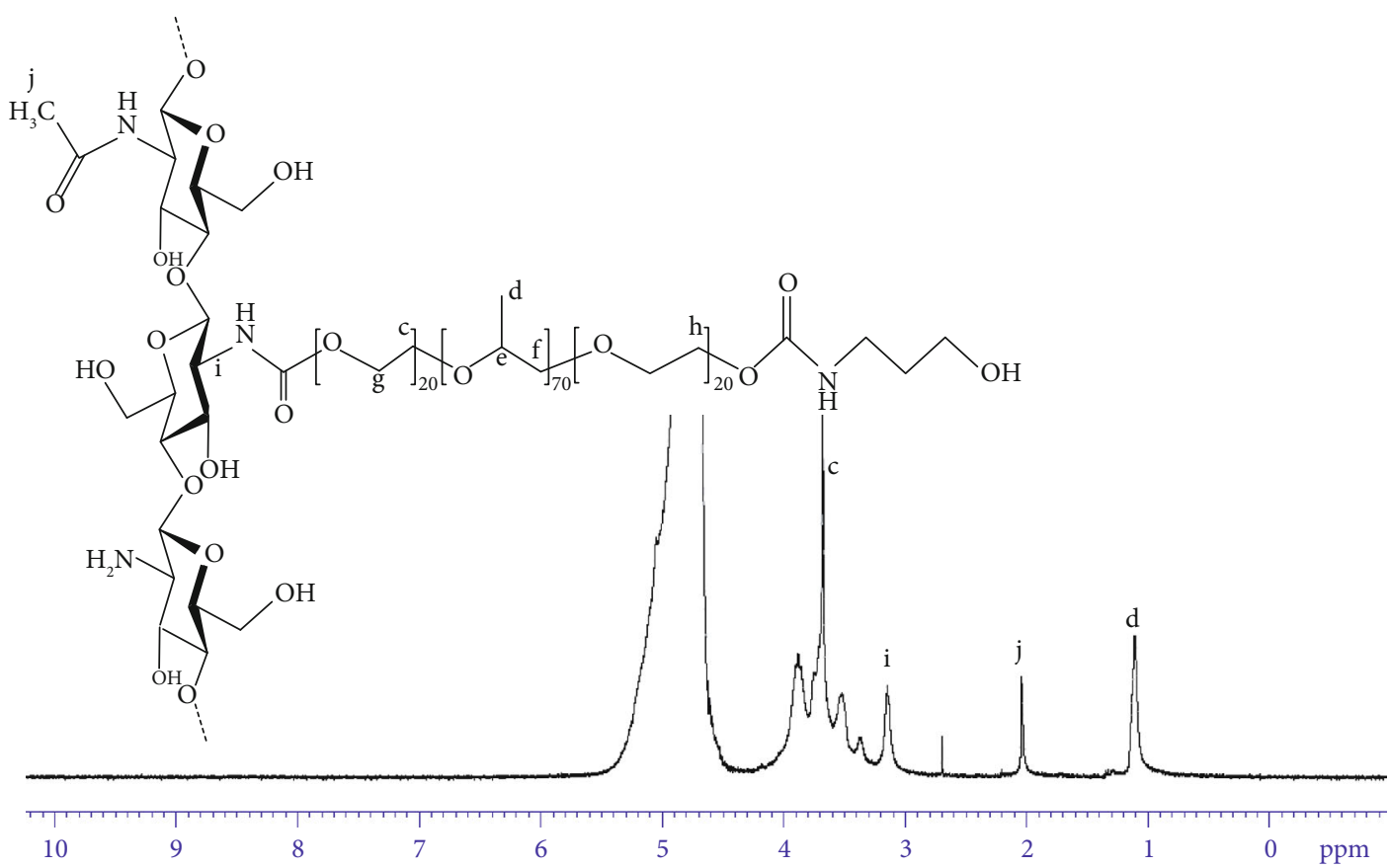

(a)

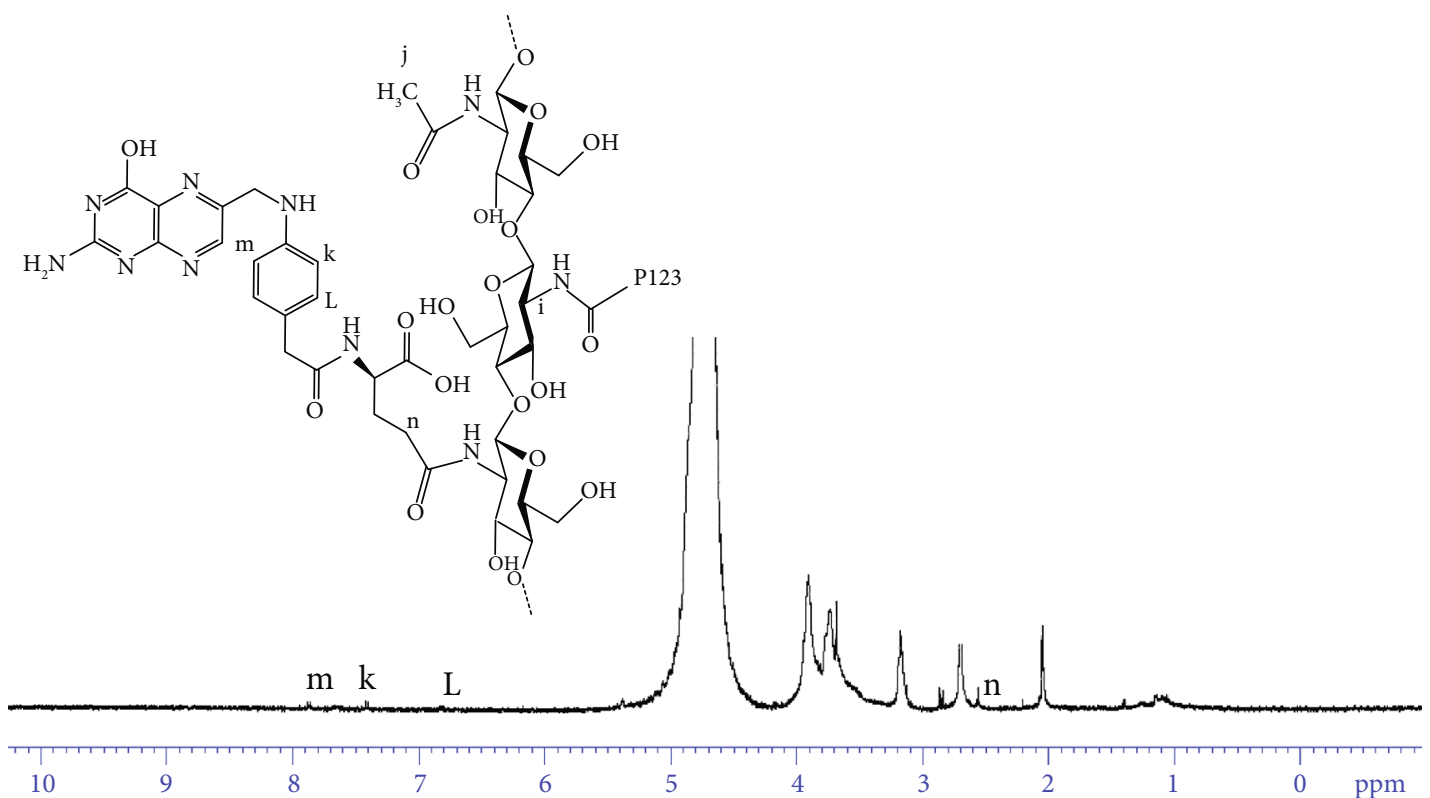

(b)

Figure 3: ${ }^{1} \mathrm{H}-\mathrm{NMR}$ spectrum of CP (a) and CP-FA (b).

$370^{\circ} \mathrm{C}$ and of 420 to $550^{\circ} \mathrm{C}$. The difference revealed the evidence for the presence of the conjugated FA moieties in a CP copolymer.

The composition of each polymer could be identified through the thermal behavior of P123 and of chitosan as well as various $\mathrm{CP}$ samples at $420^{\circ} \mathrm{C}$ at which $\mathrm{P} 123$ was fully decomposed [25]. The content of the pluronic grafted onto a chitosan chain was in the range from 73.92 to $89.90 \mathrm{wt} / \mathrm{wt} \%$ (Table 1) and reached the equilibrium of grafting reaction when the initial feeding amount of P123 was at $93.75 \%$ $(\mathrm{CP}(1: 15))$. Even with a further increase, the grafted effi- ciency of a fed P123 was nonsignificantly changed due to the steric effect of the prior P123-grafted moieties.

3.2. Characterization of Micelle and Nanogel Formation. The relationship between the $\mathrm{CMC}$ value and the hydrophobic drug loading efficiency of the micelles or nanogels was investigated in previous reports $[16,30,31]$. Moreover, the stability of the compound-loaded micelles also depends on the value of $\mathrm{CMC}$ [31]. In this study, CMC values were determined using the iodine method [30]. Solubilized iodine ion is involved in the hydrophobic environment of the PPO 


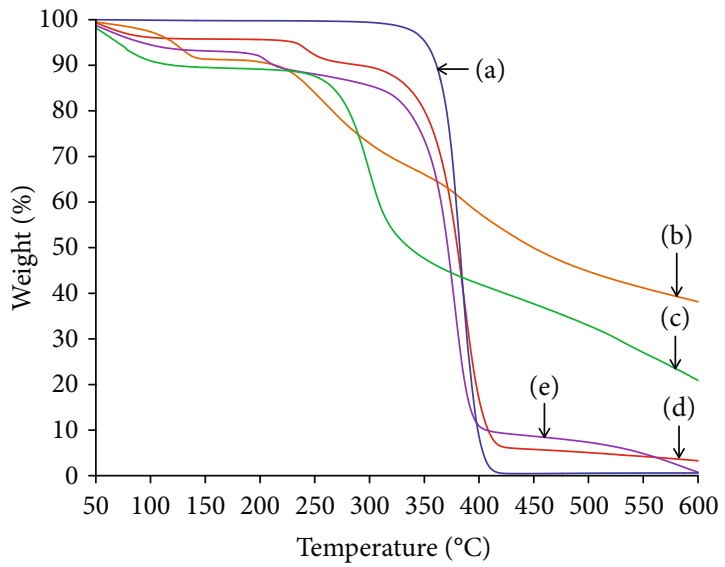

Figure 4: TGA of P123 (a), folic acid (b), chitosan (c), CP(1:10) (d), and CP-FA (e).

TABle 1: Composition and grafted degree of the CP copolymers calculated from TGA curves.

\begin{tabular}{lcccc}
\hline Formulations & $\begin{array}{c}\text { Chitosan } \\
\text { content } \\
(\%)\end{array}$ & $\begin{array}{c}\text { Pluronic } \\
\text { content } \\
(\%)\end{array}$ & $\begin{array}{c}\text { Grafted ratio } \\
\text { chitosan:P123 }\end{array}$ & $\begin{array}{c}\text { Grafted } \\
\text { degree } \\
(\%)\end{array}$ \\
\hline $\mathrm{CP}(1: 5)$ & 26.08 & 73.92 & $1: 3.5$ & 56.69 \\
$\mathrm{CP}(1: 10)$ & 16.05 & 83.95 & $1: 5.09$ & 52.29 \\
$\mathrm{CP}(1: 15)$ & 11.91 & 88.09 & $1: 7.39$ & 49.31 \\
$\mathrm{CP}(1: 20)$ & 10.01 & 89.90 & $1: 8.98$ & 44.51 \\
\hline
\end{tabular}

blocks, causing the conversion of $I_{3}^{-}$into $I_{2}$ from the residual $\mathrm{KI}$ in the solution [30]. Under CMC values, surfactants exhibit as unimers rather than in micelles; thus, no conversion of $I_{3}^{-}$into $I_{2}$ induces the constant in the absorption intensity of the mixture solution. After micelle formation, $I_{3}^{-}$is transformed into $I_{2}$ that is entrapped into the hydrophobic space of PPO blocks leading to a dramatic increase in the absorption intensity as demonstrated in Figure 5(a). The absorption intensity of $I_{2}$ had been plotted as a function of copolymer CP concentration (Figure 5(b)). Figure 5(c) indicates that the $\mathrm{CMC}$ values of various $\mathrm{CP}$ and $\mathrm{CP}-\mathrm{FA}$ formulations were much lower than that of pure P123. An increasing amount of the grafted P123 or a high content of P123 in the CP copolymer contributed to enlarging the CMC value. Many previous studies have also shown that conjugation with polysaccharides leads to a reduction of the CMC value of pluronics $[6,32]$. Chitosan attacks pluronic chains and shields the hydrophobic blocks from interaction with water reducing the interfacial free energy of the polymer-water system. Minimizing interfacial free energy helps to reduce the concentration at which micelle formation begins [33]. Hence, the lower CMC values were expected.

Along with the changing CMC as the amount of the grafted P123 increased, the size distribution of the pluronic-based nanogels was also reduced as the P123 content increased in the $\mathrm{CP}$ copolymer because a compacted core structure occurred when a hydrophobic force is predominant (Table 2). The zeta potentials of CP and CP-FA formulation micelles indicated that the surface charges were positive as a result of the $\mathrm{NH}^{3+}$ groups of chitosan. In the case of the higher grafted ratio, a decrement in zeta potential could be seen due to the loss of the functional $\mathrm{NH}_{2}$ groups of chitosan by binding to P123. The high zeta potentials of CP nanogels imply that the core-shell structure was produced in high stability forms.

Transmission electron microscopy (TEM) images of the P123, CP, and CP-FA nanogels (Figures 6(a1)-6(f1)) show that the nanoparticles (P123, $\mathrm{CP}(1: 5), \mathrm{CP}(1: 10)$, $\mathrm{CP}(1: 15), \mathrm{CP}(1: 20)$, and CP-FA) were in a spherical shape with a size diameter of $40.05 \pm 7.50,38.00 \pm 6.62,32.17 \pm$ $2.31,28.63 \pm 2.34,26.70 \pm 2.36$, and $24.27 \pm 2.01 \mathrm{~nm}$, respectively. However, TEM images in Figures 6(a3)-6(f3) show that the diameter of the nanoparticles are much smaller than that from DLS (Figures 6(a1)-6(f1)). This is because the basis for the measurement of the particle size of DLS is hydrodynamic diameter, which includes polymer shells and hydration layers as well as the swelling of chitosan and PEO block in a water environment leading to a larger particle size [16]. Moreover, in Figures 6(a3)-6(f3), the normalized intensity autocorrelation functions $g_{2}(\tau)$ fit to stretched exponential forms which could indicate that the nanoparticles were produced at the spherical micelles without free unimers [34]. Together with a moderate positive value of the zeta potential (Table 2), the results could confirm that the nanoparticles showed a high stability in colloidal solution without any dissolution or aggregation.

3.3. Characterization and Encapsulation Efficiency of DrugLoaded CP Nanogels. The CP with very low CMC values should select an appropriate vehicle for encapsulating a drug into the core of micelles. At concentrations above CMC values, micelles are protected by a hydrophilic outer shell that limits the attraction of hydrophobic drugs into the core of micelles when the emulsion methods are used. The thin film method seems to have more advantages for encapsulating a drug into micelles [35-37]. As shown in Figure 7(a), the highest encapsulation efficiencies (EE) could be seen in a range of Cur concentrations from 5 to $10 \%$. EE did not increase when the Cur concentration was elevated further. The $\mathrm{CP}(1: 10)$ performed with the highest EE reaching approximately $100 \%$. This copolymer also showed the highest drug loading capacity as seen in Figure 7(b). It is interesting that an increment in hydrophobic P123 moieties in $\mathrm{CP}(1: 15)$ and $\mathrm{CP}(1: 20)$ did not lead to improving the entrapment and loading capacity of hydrophobic Cur. The results offer that a positively charged chitosan backbone could have an impact on the EE and DL values via the electrostatic interaction of the protonated chitosan and partially negatively charged Cur [16]. This suggested that a high encapsulation of Cur contributed by both hydrophobic and electrostatic interactions of $\mathrm{CP}$ copolymers and the bioactive molecule. Due to a well-performed encapsulation ability, $\mathrm{CP}(1: 10)$ was selected as an appropriate platform for further studies.

For further study, $\mathrm{CP}(1: 10)$ was also investigated for the encapsulation of PTX. As shown in Table 3, the loading ability was the most effective at concentrations of $2 \mathrm{wt} / \mathrm{wt} \%$. 


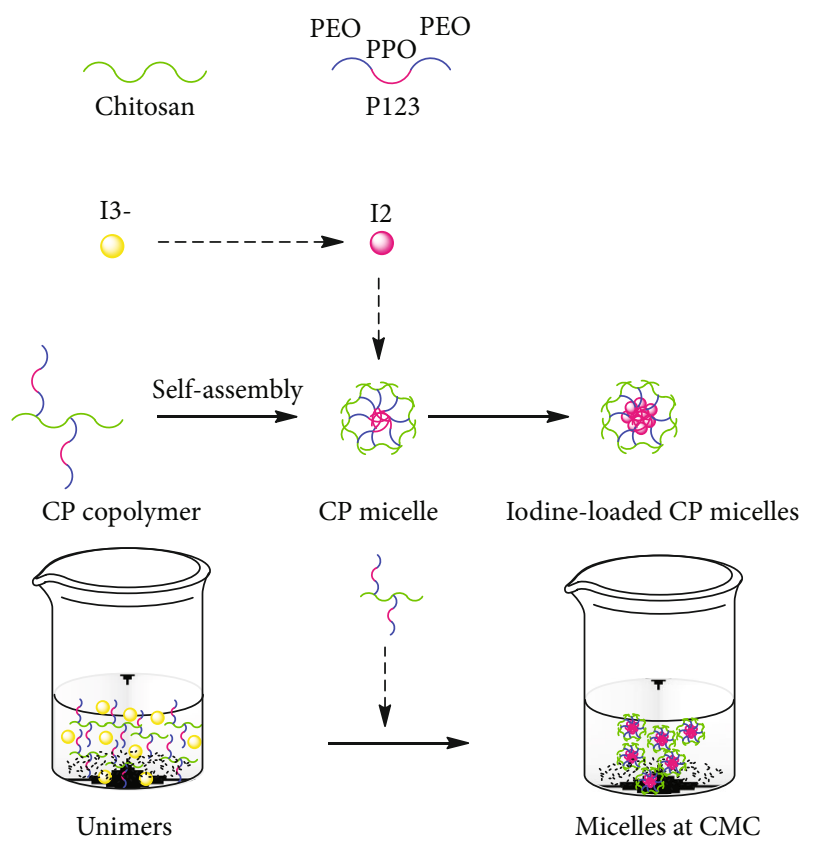

(a)

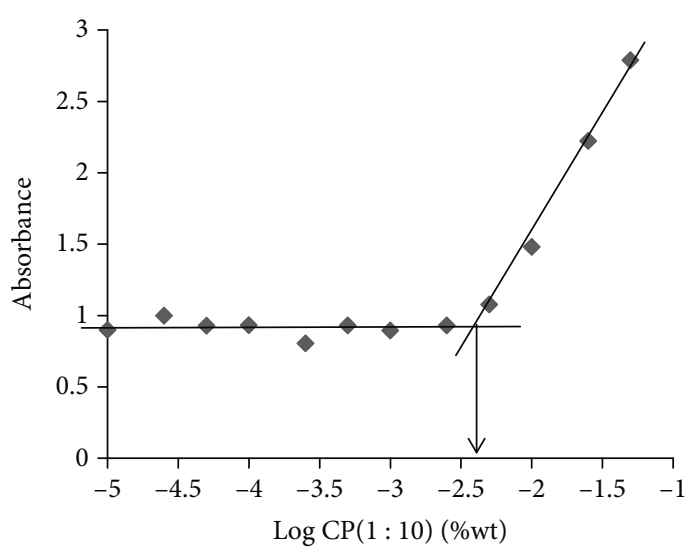

(b)

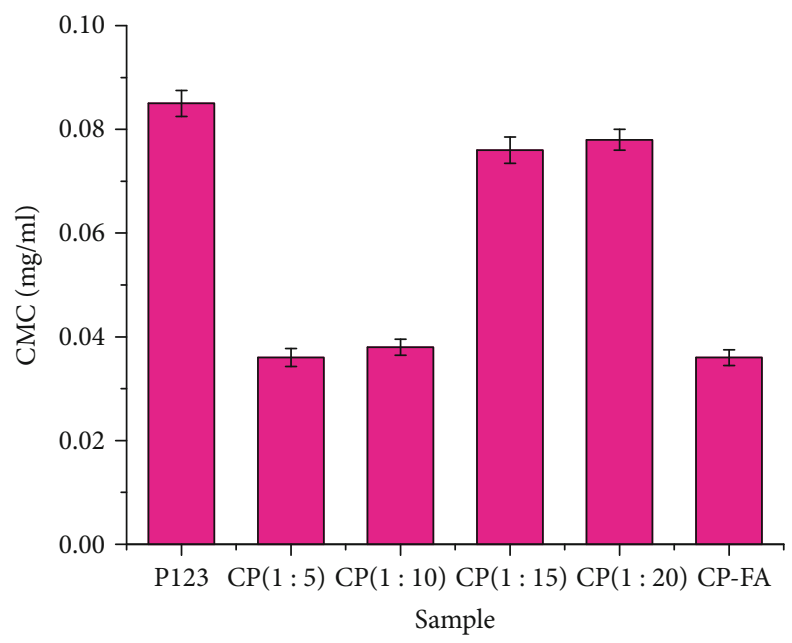

(c)

FIGURE 5: Illustration of $I_{2}$ micellization (a), plot of UV-Vis intensity of $I_{2}$ versus copolymer concentrations (b), and CMC value of various copolymers (c) $(n=3)$.

TABLE 2: DLS, zeta potentials, and CMC of P123 and various CP micelles in DI water $(n=3)$.

\begin{tabular}{lccc}
\hline Formulations & DLS $(\mathrm{nm})$ & Zeta potential $(\mathrm{mV})$ & $\mathrm{CMC}(\mathrm{mg} / \mathrm{ml})$ \\
\hline $\mathrm{P} 123$ & 104.80 & $-8.77 \pm 0.52$ & $0.085 \pm 0.003$ \\
$\mathrm{CP}(1: 5)$ & 97.20 & $60.77 \pm 1.60$ & $0.036 \pm 0.002$ \\
$\mathrm{CP}(1: 10)$ & 78.60 & $58.40 \pm 0.54$ & $0.038 \pm 0.002$ \\
$\mathrm{CP}(1: 15)$ & 69.63 & $57.00 \pm 1.10$ & $0.076 \pm 0.003$ \\
$\mathrm{CP}(1: 20)$ & 64.70 & $51.43 \pm 0.92$ & $0.078 \pm 0.002$ \\
$\mathrm{CP}-\mathrm{FA}$ & 51.10 & $39.43 \pm 3.45$ & $0.036 \pm 0.002$ \\
\hline
\end{tabular}

When the concentrations of PTX were fed at 4 and $8 \mathrm{wt} / \mathrm{wt} \%$, PTX encapsulation efficiencies decreased significantly. In our hypothesis, the PTX loading capacity was low despite a high amount of feeding. This might be due to its strong hydrophobicity as compared to Cur. The average size of the PTXloaded $\mathrm{CP}(1: 10)$ was dependent on the PTX loading amount. The mean size increased gradually in the range of the fed PTX from 1 to $8 \mathrm{wt} / \mathrm{wt} \%$. This behavior could be explained by the bulky structure of PTX resulting in an increase of the PTX-loaded nanoparticles. The zeta potential result of samples showed that all nanogels formed under the positively charged form. Regarding Cur and PTX loading in a single investigation, the $\mathrm{CP}(1: 10)$ formulation could be selected for encapsulating 10 and $2 \mathrm{wt} / \mathrm{wt} \%$, respectively. Furthermore, $\mathrm{CP}(1: 10)$ was selected to conjugate folic acid towards the fabrication of targeted delivery in inhibitory cancer cell growth.

3.4. Characterizations of Dual Cur/PTX-Loaded CP-FA. According to the optimal encapsulating content of Cur- (or PTX-) loaded CP, dual-loading PTX and Cur were at 2 and $10 \mathrm{wt} / \mathrm{wt} \%$ onto CP-FA, respectively. The results showed that the encapsulating ability was not significantly changed for each drug as seen in Table 4. In the PTX-loaded formulations, an increment of size distribution could be observed from DLS measurement. The size distribution of the drugloaded nanocarriers also followed the trend of TEM results, in which dual drug-loaded nanogels appeared in the biggest 
(a1)

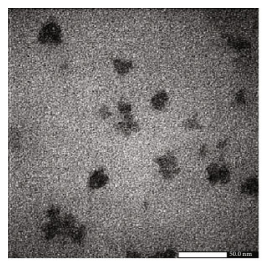

(a)

(b1)

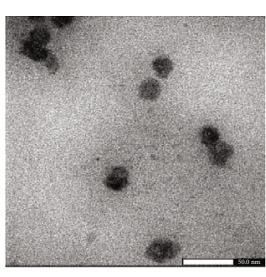

(b2)

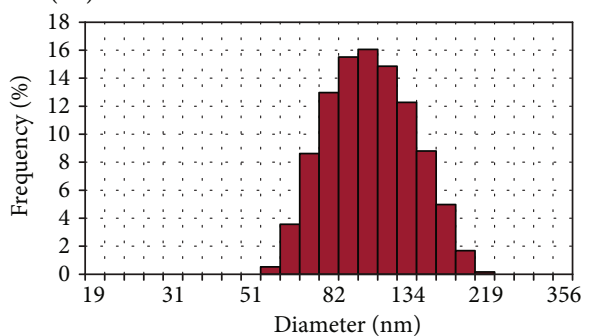

(b)

(c1)
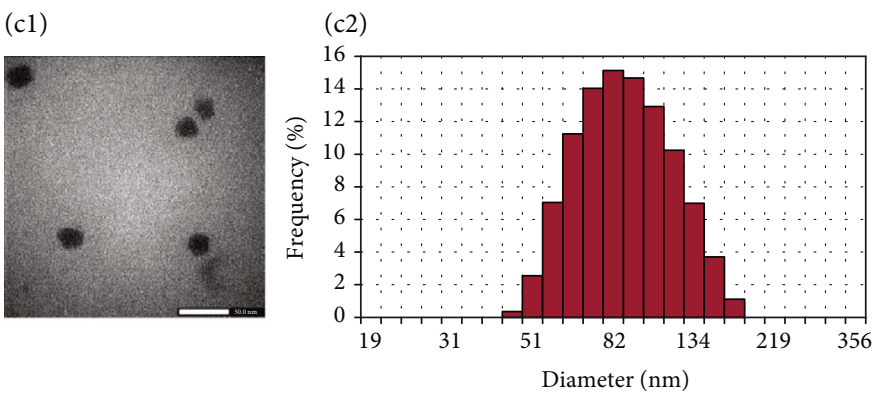

(c)

(d1)

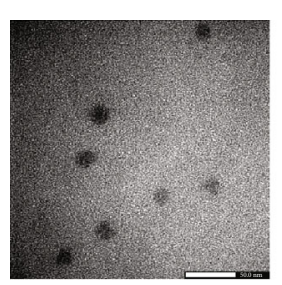

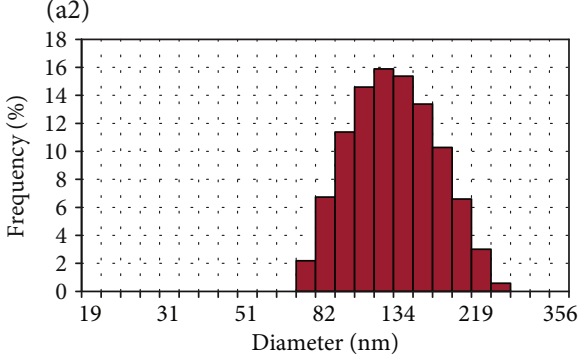

(a3)

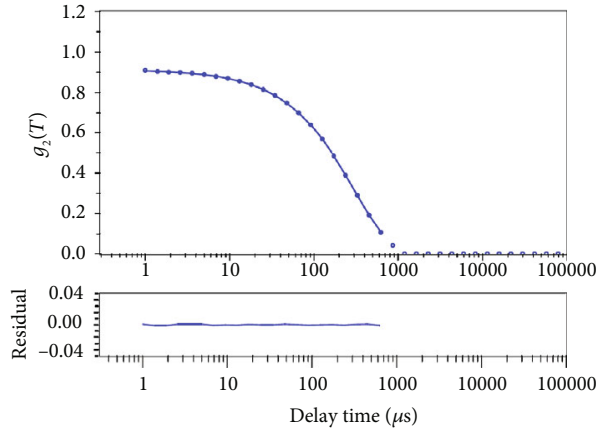

(b3)

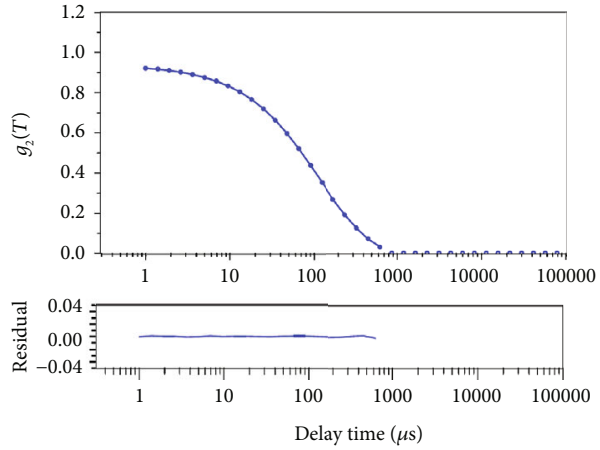

(c3)

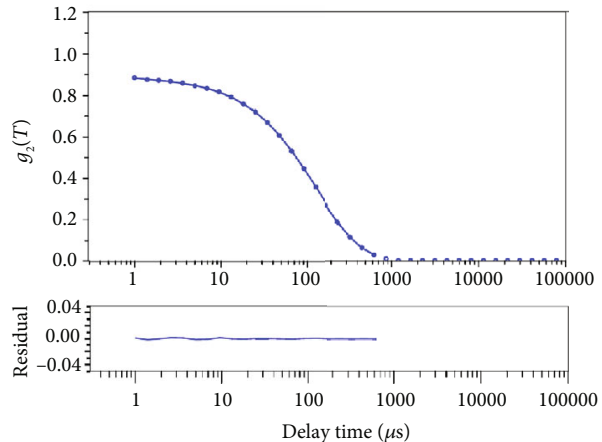

(d3)
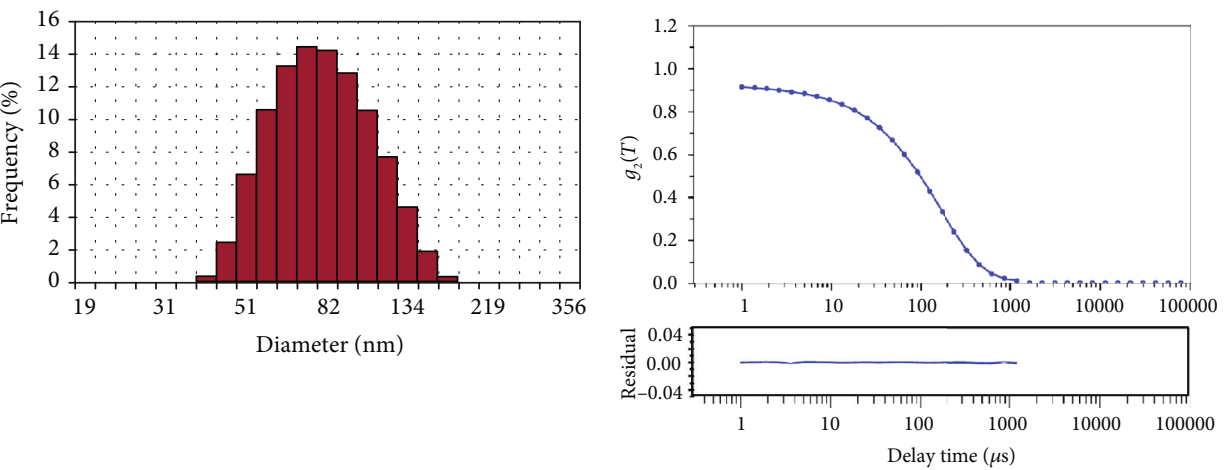

(d)

Figure 6: Continued. 
(e1)

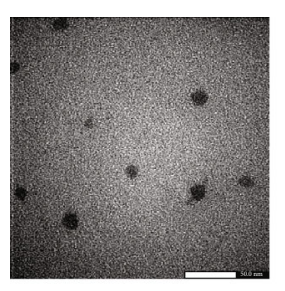

$$
\text { (e2) }
$$

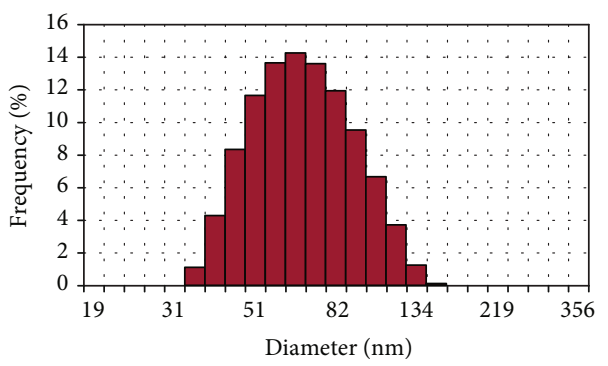

(e)

(f1)

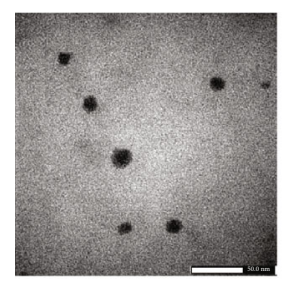

(e3)

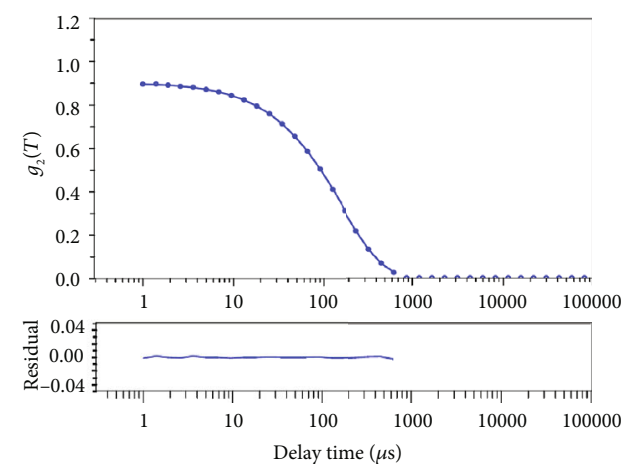

(f3)

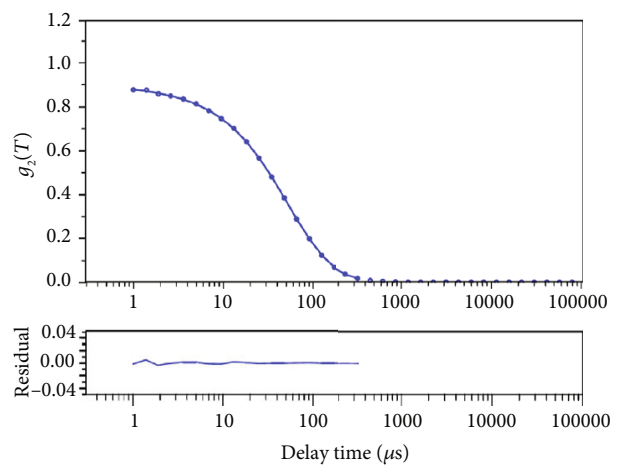

(f)

Figure 6: TEM micrographs and DLS of $\mathrm{P} 123$ (a), $\mathrm{CP}(1: 5)$ (b), $\mathrm{CP}(1: 10)$ (c), $\mathrm{CP}(1: 15)(\mathrm{d}), \mathrm{CP}(1: 20)(\mathrm{e})$, and CP-FA (f).

spherical shape as compared to single-loading CP-FA (Figure 8). These obtained results were strongly supported for discussion related to the bulky structure of PTX.

3.5. In Vitro Release Behavior. The drug release profile is a critical parameter for evaluating the effectiveness of a delivery system. The CP-FA nanogels were investigated for the release profile behavior of single and dual drugs at physiological condition ( $\mathrm{pH} 5.6$ and $\mathrm{pH}$ 7.4). In general, all drugs performed in a sustained-release manner as seen in Figure 9. The cumulative release rate of PTX from PTX/Cur-loaded CP-FA was slightly higher than that of its release rate from PTX-loaded CP-FA. The cumulative release profile of Cur from a dual PTX/Cur-loaded CP-FA was lower than that of a single-loaded CP-FA. This could be explained by a higher hydrophobic interaction of PTX or PTX/Cur with a hydrophobic domain in CP-FA resulting in the well-controlled release of the loaded PTX or PTX and Cur. The behavior is similar to that found in Nguyen et al.'s study in which a hydrophobic drug had a slightly slower release behavior from a higher hydrophobic platform [25]. In addition, Figures 9(a) and 9(b) show the release profile of PTX and Cur affected by the incubated media that exhibited a faster release rate at $\mathrm{pH} 5.6$ as compared to $\mathrm{pH}$ 7.4. For instance, the PTX cumulative release rates increased from $16.08 \%$ at $\mathrm{pH} 7.4$ to $23.80 \%$ at pH 5.6 for PTX/Cur-CP-FA after $48 \mathrm{~h}$. At the same time, the Cur cumulative release rates performed in a similar behavior. This pH-dependent PTX and Cur release could be explained by the protonation of the CP-FA platform resulting in the swelling behavior of nanogels that lead to a higher release rate of PTX and Cur. The sustained and pH-dependent release of PTX and Cur could be significant for enhancing drug accumulation at the tumor site and for achieving intracellular release of the combined anticancer drugs.

3.5.1. In Vitro Cytotoxicity. In recent years, there have been several reports on the synergistic effect of phytocompounds and anticancer drugs in clinical treatment $[38,39]$. A combination of the effect in nanocarriers has also received much attention [27-29]. In this study, CP-FA, free PTX, PTXCP-FA, PTX/Cur-CP-FA, and Cur-CP-FA were evaluated for inhibitory activity against MCF-7 cancer cell growth using a sulforhodamine B (SRB) colorimetric assay. After $48 \mathrm{~h}$ of incubation, the result showed that CP-FA exhibited an inhibitory value at $18 \pm 0.5 \%$ at $100 \mu \mathrm{g} / \mathrm{ml}$ of screening concentration. Free PTX exhibited very high cytotoxicity with an IC50 value of $4.04 \pm 1.02 \mathrm{nM}$. However, after loading in a CP-FA nanogel, the cytotoxicity of PTX was significantly reduced to a IC50 value of $8.20 \pm 1.41 \mathrm{nM}$. In the case of a single-loaded Cur, Cur-CP-FA performed a low anticancer activity (IC50 $=13.00 \pm 1.42 \mu \mathrm{M})$. It is highly attractive that the dual-drug-loaded nanogel (PTX/Cur-CP-FA) significantly enhanced toxicity against MCF-7 cancer cell growth (IC50 $=5.74 \pm 0.23 \mathrm{nM})$ as compared to a single-PTXloaded platform. Free PTX exhibited a better cytotoxic activity with a lower IC50 value than PTX-CP-FA and PTX/Cur- 


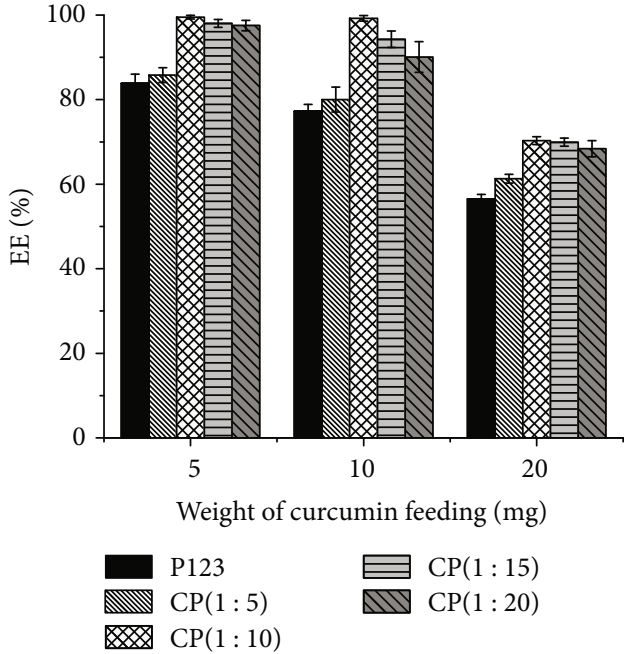

(a)

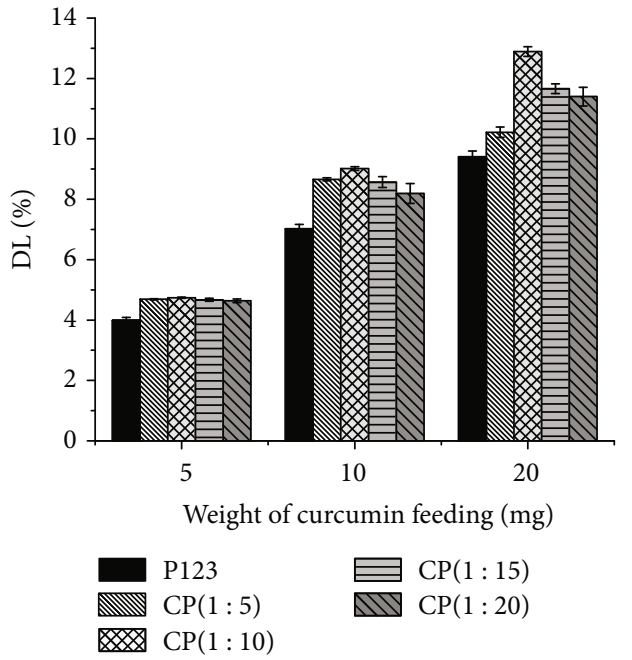

(b)

FIgURE 7: Encapsulation efficiencies (a) and drug loading (b) of various Cur-loaded CP formulations $(n=3)$.

TABLE 3: EE, DL, DLS, and zeta potentials of various PTX-loaded CP(1:10) formulations $(n=3)$.

\begin{tabular}{lcccccc}
\hline Sample & CP $(\mathrm{mg})$ & PTX $(\mathrm{mg})$ & EE $(\%)$ & DL $(\%)$ & Size $(\mathrm{DLS}, \mathrm{nm})$ & Zeta potentials $(\mathrm{mV})$ \\
\hline 1 & 100 & 1 & $97.5 \pm 0.48$ & $0.97 \pm 0.01$ & $49.60 \pm 1.10$ & $36.27 \pm 1.60$ \\
2 & 100 & 2 & $97.65 \pm 1.35$ & $1.91 \pm 0.03$ & $52.80 \pm 1.85$ & $34.03 \pm 0.86$ \\
3 & 100 & 4 & $61.07 \pm 0.40$ & $2.35 \pm 0.02$ & $53.90 \pm 0.57$ & $31.23 \pm 0.09$ \\
4 & 100 & 8 & $41.29 \pm 0.59$ & $3.06 \pm 0.04$ & $63.47 \pm 0.60$ & $24.30 \pm 1.02$ \\
\hline
\end{tabular}

TABLe 4: EE, DL, and DLS of various Cur and PTX-loaded CP-FA formulations $(n=3)$.

\begin{tabular}{|c|c|c|c|c|c|c|}
\hline Samples & CP-FA (mg) & PTX (mg) & Cur (mg) & $\mathrm{EE}(\%)$ & DL (\%) & DLS (nm) \\
\hline 1 & 100 & 2 & - & $98.21 \pm 0.49$ & $1.93 \pm 0.01$ & $53.93 \pm 0.65$ \\
\hline 2 & 100 & 2 & 10 & $\begin{array}{c}98.63 \pm 0.42(\mathrm{PTX}) \\
97.82 \pm 0.48 \text { (Cur) }\end{array}$ & $\begin{array}{l}1.76 \pm 0.01(\mathrm{PTX}) \\
8.83 \pm 0.01 \text { (Cur) }\end{array}$ & $60.57 \pm 0.21$ \\
\hline 3 & 100 & - & 10 & $99.13 \pm 0.35$ & $9.01 \pm 0.01$ & $44.83 \pm 0.25$ \\
\hline
\end{tabular}

CP-FA, owing to a different amount of PTX present in the cytoplasm of MCF-7 cells. According to the release profiles, only $19.46 \%$ or $13.05 \%$ PTX was released from CP-FA at pH 5.6 and $\mathrm{pH} 7.4$ after $48 \mathrm{~h}$, respectively. This means that the concentration of PTX from CP-FA in MCF-7 cells is lower than that of free PTX in MCF-7 cells. Moreover, free PTX could readily deliver into the cytosol via passive diffusion and be quickly effective, whereas the drug-loaded platforms were mainly taken up by cells via the endocytic pathway (due to the charge surface of vesicles) and then the drugs released PTX in a sustained manner resulting in certain cytotoxicity. The results also agreed with some previous reports in which a free drug is more cytotoxic than a drugloaded platform [40, 41]. As seen in Figure 10, the dualloading formulation exhibits a higher activity than that of a single-loading sample (PTX-CP-FA) starting from $1.17 \mathrm{nM}$ of PTX-loaded concentration. These results could confirm a synergistic effect of Cur and PTX in the new CP-FA nanogel. The work should also be studied further to clarify the role of the FA targeting ligand in the specific delivery of Cur and PTX towards cancer cells.

\section{Conclusions}

In this paper, we successfully developed a series of differently amphiphilic-grafted chitosan-P123 copolymers characterized towards drug delivery. The current results indicated that $\mathrm{CP}(1: 10)$ performs highly efficiently in Cur and PTX drug loading. The CP formulation was utilized for conjugating the FA-targeted ligand. The CP-FA nanogels exhibited great behavior with a hydrophobic drug encapsulation efficiency that was up to $98.63 \pm 0.42$ of PTX and 97.82 \pm 0.48 of Cur. The size of the dual-loaded CP-FA nanogels were distributed at $60.57 \pm 0.21 \mathrm{~nm}$ which may be appropriate for cell 


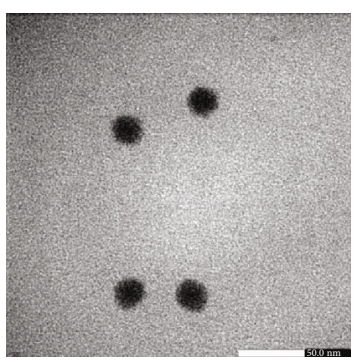

(a)

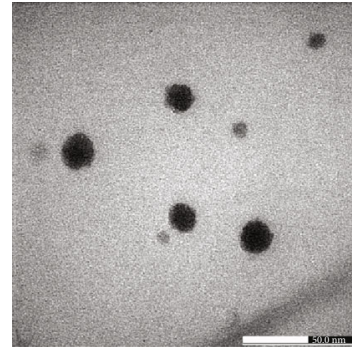

(b)

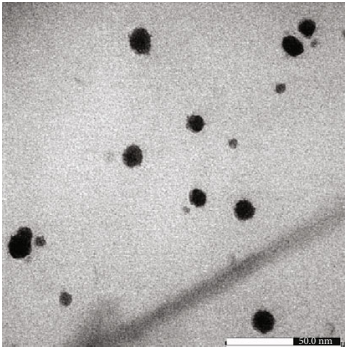

(c)

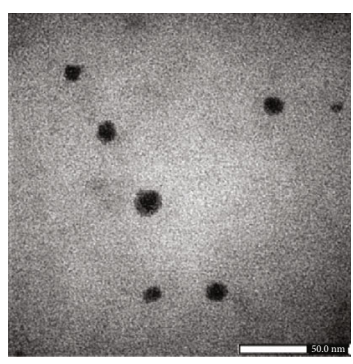

(d)

Figure 8: TEM images of PTX-CP-FA (a), PTX/Cur-CP-FA (b), Cur-CP-FA (c), and CP-FA nanogels.

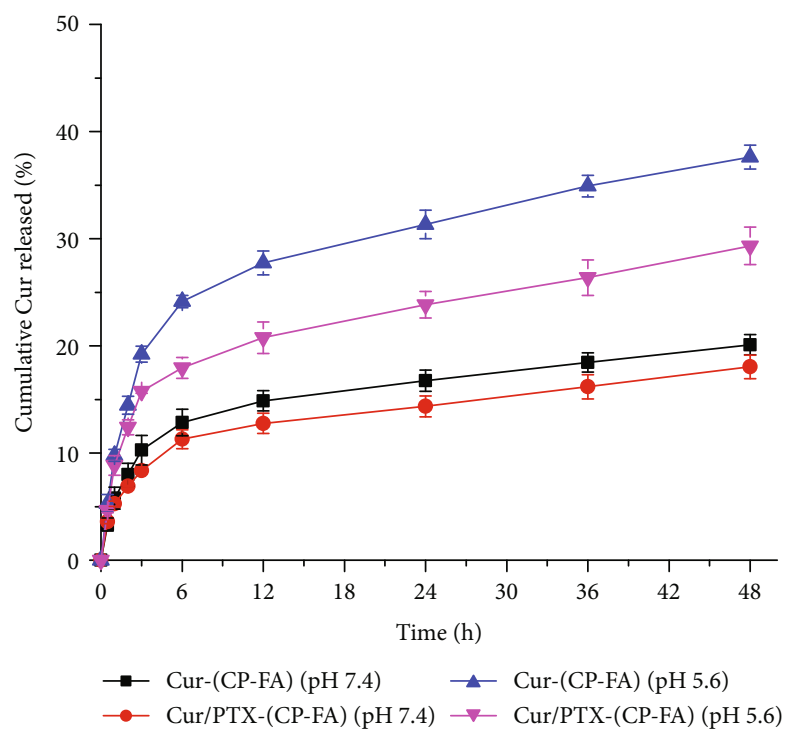

(a)

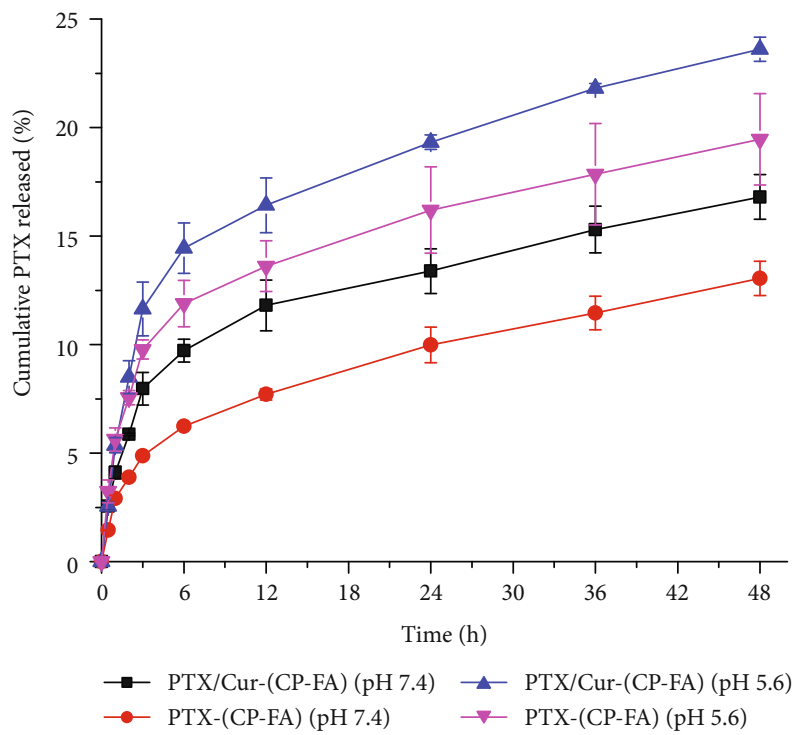

(b)

Figure 9: In vitro release of Cur (a) and PTX (b) from CP-FA at various pH values (7.4 and 5.6) $(n=3)$.

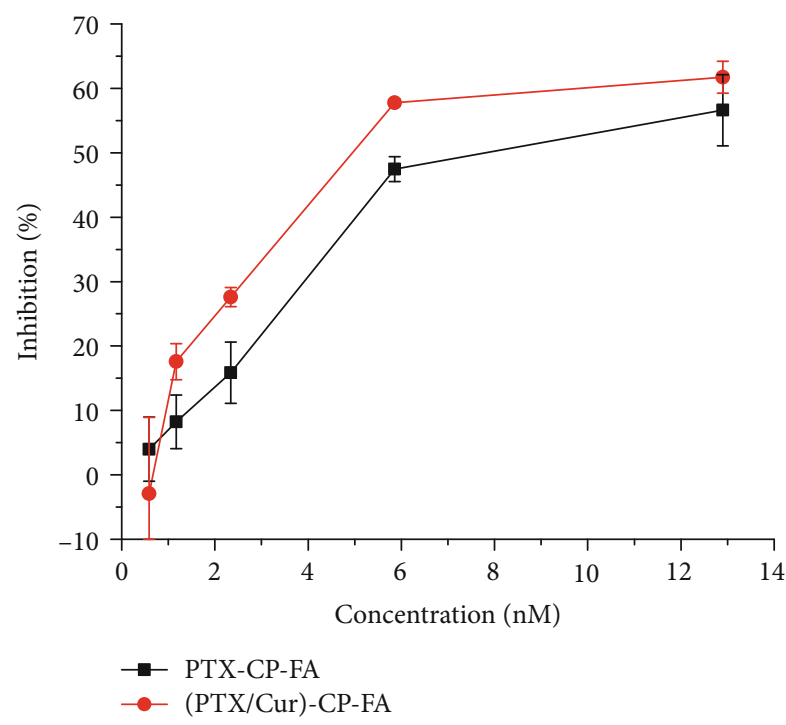

Figure 10: Cytotoxicity of PTX-CP-FA and PTX/Cur-CP-FA on MCF-7 cancer cells $(n=3)$. endocytosis. The release profile behavior of drug-loaded CPFA depended on $\mathrm{pH}$. Moreover, the combination of the dual drugs in the CP-FA delivery platform could offer a synergistic effect against cancer cell growth. The dual-drug-loaded nanogel (PTX/Cur-CP-FA) significantly enhanced toxicity against MCF-7 cancer cell growth (IC50 $=5.74 \pm 0.23 \mathrm{nM}$ ) that was better than that of the PTX-loaded platform $(\mathrm{IC} 50=8.20 \pm 1.41 \mathrm{nM})$. Regarding these obtained results, the work exhibited some positive facets that could pave a way for further studies to clarify the effectiveness of PTX/Cur-loaded CP-FA in the specific delivery for cancer treatment in vivo.

\section{Data Availability}

The data used to support the findings of this study are available from the corresponding author upon request.

\section{Conflicts of Interest}

The authors declare that they have no conflicts of interest. 


\section{Acknowledgments}

This work was funded by the Vietnam National Foundation for Science and Technology Development (NAFOSTED) under grant number 104.02-2017.60.

\section{References}

[1] J. H. Choi, Y. K. Joung, J. W. Bae, J. W. Choi, T. N. Quyen, and K. D. Park, "Self-assembled nanogel of pluronic-conjugated heparin as a versatile drug nanocarrier," Macromolecular Research, vol. 19, no. 2, pp. 180-188, 2011.

[2] P. N. Le, C. K. Huynh, and N. Q. Tran, "Advances in thermosensitive polymer-grafted platforms for biomedical applications," Materials Science and Engineering: C, vol. 92, pp. 1016-1030, 2018.

[3] L. Han, J. Guo, L. Zhang, Q. Wang, and X. Fang, "Pharmacokinetics and biodistribution of polymeric micelles of paclitaxel with pluronic P123," Acta Pharmacologica Sinica, vol. 27, no. 6, pp. 747-753, 2006.

[4] K. M. Park, J. W. Bae, Y. K. Joung, J. W. Shin, and K. D. Park, "Nanoaggregate of thermosensitive chitosan-pluronic for sustained release of hydrophobic drug," Colloids and Surfaces B: Biointerfaces, vol. 63, no. 1, pp. 1-6, 2008.

[5] K. M. Park, Y. K. Joung, K. D. Park, S. Y. Lee, and M. C. Lee, "RGD-conjugated chitosan-pluronic hydrogels as a cell supported scaffold for articular cartilage regeneration," Macromolecular Research, vol. 16, no. 6, pp. 517-523, 2008.

[6] X. Tao, Y. Li, Q. Hu et al., "Preparation and drug release study of novel nanopharmaceuticals with polysorbate 80 surface adsorption," Journal of Nanomaterials, vol. 2018, Article ID 4718045, 11 pages, 2018

[7] T. Fan, X. Yu, B. Shen, and L. Sun, "Peptide self-assembled nanostructures for drug delivery applications," Journal of Nanomaterials, vol. 2017, Article ID 4562474, 16 pages, 2017.

[8] H. Lu, J. Wang, T. Wang, J. Zhong, Y. Bao, and H. Hao, "Recent progress on nanostructures for drug delivery applications," Journal of Nanomaterials, vol. 2016, Article ID 5762431, 12 pages, 2016.

[9] T. Miao, J. Wang, Y. Zeng, G. Liu, and X. Chen, "Polysaccharide-based controlled release systems for therapeutics delivery and tissue engineering: from bench to bedside," Advanced Science, vol. 5, no. 4, article 1700513, 2018.

[10] P. Eslami, F. Rossi, and S. Fedeli, "Hybrid nanogels: stealth and biocompatible structures for drug delivery applications," Pharmaceutics, vol. 11, no. 2, p. 71, 2019.

[11] Z. Li, Z. Zhang, K. L. Liu, X. Ni, and J. Li, "Biodegradable hyperbranched amphiphilic polyurethane multiblock copolymers consisting of poly (propylene glycol), poly (ethylene glycol), and polycaprolactone as in situ thermogels," Biomacromolecules, vol. 13, no. 12, pp. 3977-3989, 2012.

[12] A. H. Shan, L. Jiang, and Z. Li, "Biodegradable polyester thermogelling system as emerging materials for therapeutic applications," Macromolecular Materials and Engineering, vol. 303, no. 5, article 1700656, 2018.

[13] A. V. Kabanov, P. Lemieux, S. Vinogradov, and V. Alakhov, "Pluronic ${ }^{\circledast}$ block copolymers: novel functional molecules for gene therapy," Advanced Drug Delivery Reviews, vol. 54, no. 2, pp. 223-233, 2002.

[14] Z. Liu, D. Liu, L. Wang, J. Zhang, and N. Zhang, "Docetaxelloaded pluronic p123 polymeric micelles: in Vitro and in Vivo evaluation," International Journal of Molecular Sciences, vol. 12, no. 3, pp. 1684-1696, 2011.

[15] L. Zhao, J. du, Y. Duan et al., "Curcumin loaded mixed micelles composed of Pluronic P123 and F68: Preparation, optimization and in vitro characterization," Colloids and Surfaces B: Biointerfaces, vol. 97, pp. 101-108, 2012.

[16] L. H. Dang, M. T. Vu, J. Chen et al., "Effect of ultrasonication on self-assembled nanostructures formed by amphiphilic positive-charged copolymers and negative-charged drug," ACS Omega, vol. 4, no. 3, pp. 4540-4552, 2019.

[17] Z. Wei, J. Hao, S. Yuan et al., "Paclitaxel-loaded pluronic P123/F127 mixed polymeric micelles: formulation, optimization and in vitro characterization," International Journal of Pharmaceutics, vol. 376, no. 1-2, pp. 176-185, 2009.

[18] W. Zhang, Y. Shi, Y. Chen et al., "Enhanced antitumor efficacy by paclitaxel-loaded pluronic P123/F127 mixed micelles against non-small cell lung cancer based on passive tumor targeting and modulation of drug resistance," European Journal of Pharmaceutics and Biopharmaceutics, vol. 75, no. 3, pp. 341-353, 2010.

[19] J. I. Lee, H. S. Kim, and H. S. Yoo, "DNA nanogels composed of chitosan and pluronic with thermo-sensitive and photocrosslinking properties," International Journal of Pharmaceutics, vol. 373, no. 1-2, pp. 93-99, 2009.

[20] J. Y. Kim, W. I. Choi, Y. H. Kim et al., "In-vivo tumor targeting of pluronic-based nano-carriers," Journal of Controlled Release, vol. 147, no. 1, pp. 109-117, 2010.

[21] T. B. T. Nguyen, L. H. Dang, T. T. T. Nguyen et al., "Green processing of thermosensitive nanocurcuminencapsulated chitosan hydrogel towards biomedical application," Green Processing and Synthesis, vol. 5, no. 6, pp. 511-520, 2016.

[22] V. Kumar, P. K. Gupta, V. K. Pawar et al., "In-Vitro and InVivo studies on novel chitosan-g-pluronic F-127 copolymer based nanocarrier of amphotericin B for improved antifungal activity," Journal of Biomaterials and Tissue Engineering, vol. 4, no. 3, pp. 210-216, 2014.

[23] L. H. Dang, T. H. Nguyen, H. L. B. Tran, V. N. Doan, and N. Q. Tran, "Injectable nanocurcumin-formulated chitosan-gpluronic hydrogel exhibiting a great potential for burn treatment," Journal of Healthcare Engineering, vol. 2018, Article ID 5754890, 14 pages, 2018.

[24] N.-A. N. Tong, T. P. Nguyen, N. Cuu Khoa, and N. Q. Tran, "Aquated cisplatin and heparin-pluronic nanocomplexes exhibiting sustainable release of active platinum compound and NCI-H460 lung cancer cell antiproliferation," Journal of Biomaterials Science, Polymer Edition, vol. 27, no. 8, pp. 709720, 2016.

[25] N. Nguyen, N. Nguyen, N. Tran et al., "Synergic activity against MCF-7 breast cancer cell growth of nanocurcuminencapsulated and cisplatin-complexed nanogels," Molecules, vol. 23, no. 12, p. 3347, 2018.

[26] Y. Zhan, Y. Chen, R. Liu, H. Zhang, and Y. Zhang, "Potentiation of paclitaxel activity by curcumin in human breast cancer cell by modulating apoptosis and inhibiting EGFR signaling," Archives of Pharmacal Research, vol. 37, no. 8, pp. 10861095,2014

[27] X. Gao, B. Wang, Q. Wu et al., "Combined delivery and anticancer activity of paclitaxel and curcumin using polymeric micelles," Journal of Biomedical Nanotechnology, vol. 11, no. 4, pp. 578-589, 2015. 
[28] Z. Liu, Y. Y. Zhu, Z. Y. Li, and S. Q. Ning, "Evaluation of the efficacy of paclitaxel with curcumin combination in ovarian cancer cells," Oncology Letters, vol. 12, no. 5, pp. 3944-3948, 2016.

[29] Q. Yao, D. C. Gutierrez, N. H. Hoang et al., "Efficient codelivery of paclitaxel and curcumin by novel bottlebrush copolymer-based micelles," Molecular Pharmaceutics, vol. 14, no. 7, pp. 2378-2389, 2017.

[30] S. K. Hait and S. P. Moulik, "Determination of critical micelle concentration (CMC) of nonionic surfactants by donor-acceptor interaction with lodine and correlation of CMC with hydrophile-lipophile balance and other parameters of the surfactants," Journal of Surfactants and Detergents, vol. 4, no. 3, pp. 303-309, 2001.

[31] B. S. M. Zadeh, G. Esfahani, and A. Salimi, "Permeability of ciprofloxacin-loaded polymeric micelles including ginsenoside as P-glycoprotein inhibitor through a Caco-2 Cells monolayer as an intestinal absorption model," Molecules, vol. 23, no. 8, article 1904, 2018.

[32] T. T. C. Nguyen, C. K. Nguyen, T. H. Nguyen, and N. Q. Tran, "Highly lipophilic pluronics-conjugated polyamidoamine dendrimer nanocarriers as potential delivery system for hydrophobic drugs," Materials Science and Engineering: C, vol. 70, Part 2, pp. 992-999, 2017.

[33] S. C. Owen, D. P. Y. Chan, and M. S. Shoichet, "Polymeric micelle stability," Nano Today, vol. 7, no. 1, pp. 53-65, 2012.

[34] R. Basak and R. Bandyopadhyay, "Encapsulation of hydrophobic drugs in pluronic F127 micelles: effects of drug hydrophobicity, solution temperature, and $\mathrm{pH}$," Langmuir, vol. 29, no. 13, pp. 4350-4356, 2013.

[35] C. P. Reis, R. J. Neufeld, A. J. Ribeiro, and F. Veiga, "Nanoencapsulation I. Methods for preparation of drug-loaded polymeric nanoparticles," Nanomedicine: Nanotechnology, Biology and Medicine, vol. 2, no. 1, pp. 8-21, 2016.

[36] C. E. Mora-Huertas, H. Fessi, and A. Elaissari, "Polymer-based nanocapsules for drug delivery," International Journal of Pharmaceutics, vol. 385, no. 1-2, pp. 113-142, 2010.

[37] U. Wais, A. W. Jackson, T. He, and H. Zhang, "Nanoformulation and encapsulation approaches for poorly water-soluble drug nanoparticles," Nanoscale, vol. 8, no. 4, pp. 1746-1769, 2016.

[38] N. M. Yunos, P. Beale, J. Q. Yu, and F. Huq, "Synergism from the combination of oxaliplatin with selected phytochemicals in human ovarian cancer cell lines," Anticancer Research, vol. 31, no. 12 , pp. 4283-4289, 2011.

[39] A. Hosseini and A. Ghorbani, "Cancer therapy with phytochemicals: evidence from clinical studies," Avicenna Journal of Phytomedicine, vol. 5, no. 2, pp. 84-97, 2015.

[40] D. Zhu, S. Wu, C. Hu et al., "Folate-targeted polymersomes loaded with both paclitaxel and doxorubicin for the combination chemotherapy of hepatocellular carcinoma," Acta Biomaterialia, vol. 58, pp. 399-412, 2018.

[41] S. Lv, Z. Tang, M. Li et al., "Co-delivery of doxorubicin and paclitaxel by PEG-polypeptide nanovehicle for the treatment of non-small cell lung cancer," Biomaterials, vol. 35, no. 23, pp. 6118-6129, 2014. 


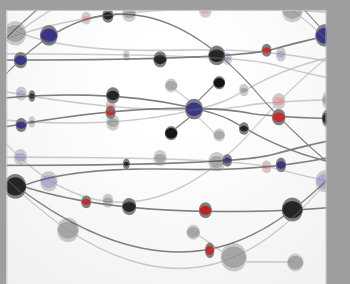

The Scientific World Journal
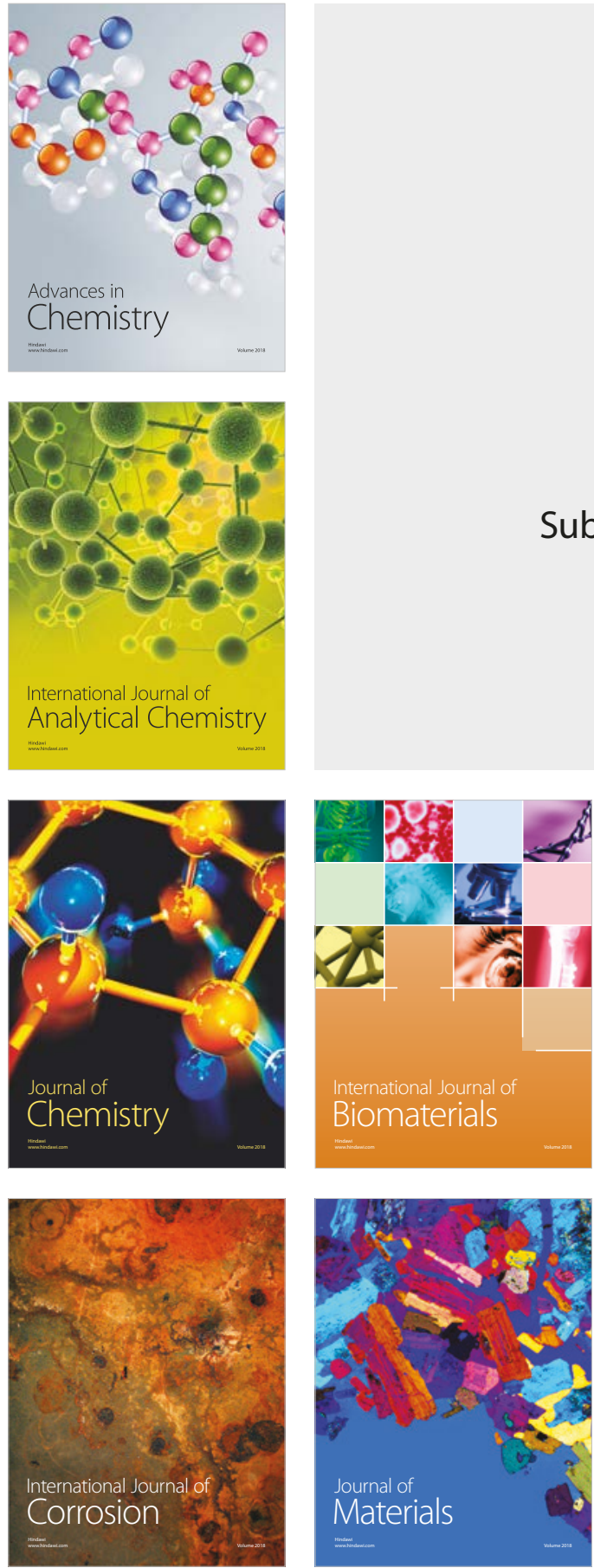

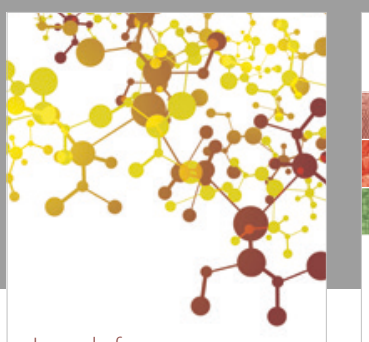

Journal of

Applied Chemistry
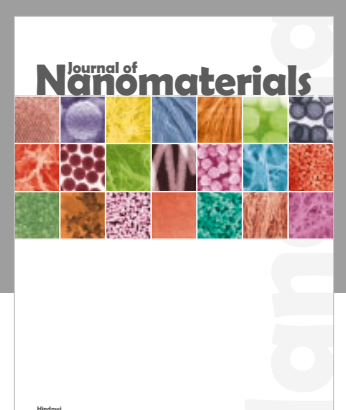

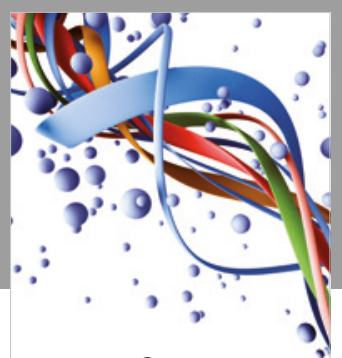

Scientifica

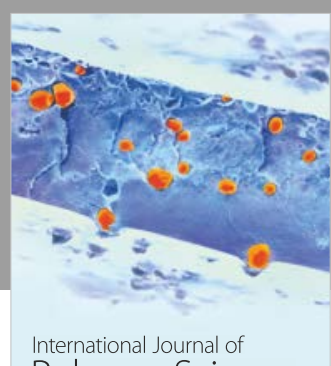

Polymer Science

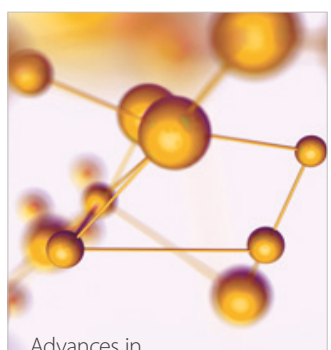

Physical Chemistry
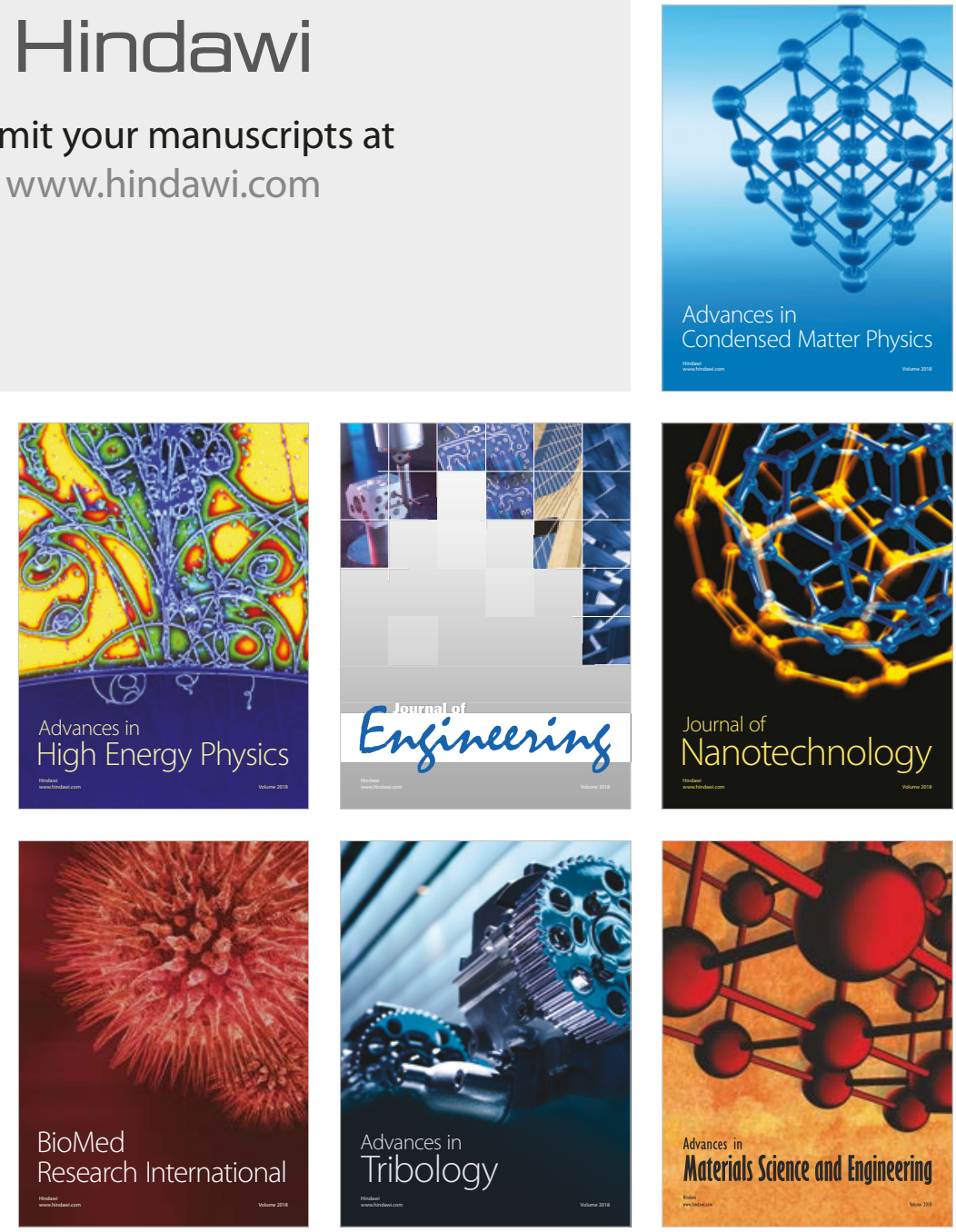